\title{
Capturing the Flexibility of a Protein-Ligand Complex: Binding Free Energies from Different Enhanced Sampling Techniques
}

\author{
Sebastian Wingbermühle* and Lars V. Schäfer* \\ Theoretical Chemistry, Ruhr University Bochum, D-44780 Bochum, Germany \\ E-mail: Sebastian.Wingbermuehle@rub.de; Lars.Schaefer@rub.de
}

\section{Simulation Details}

In all simulations discussed here, except for the exploratory standard MD simulations presented in Fig. S1, the potential energy of the protein was given by the AMBER99SB*-ILDNP force field ${ }^{1-5}$, and the water molecules were represented by the TIP4P $/ 2005$ model $^{6}$. All simulations were run using the program package $G R O M A C S^{7-13}$. The system preparation and the Umbrella Sampling (US) ${ }^{14-16}$ simulations were performed with version 5.0.6; the Bias Exchange Umbrella Sampling (BEUS, or replica-exchange umbrella sampling) ${ }^{17}$, Replica Exchange with Solute Tempering 2 (REST2) ${ }^{18-20}$, and well-tempered Metadynamics (MTD) ${ }^{21}$ simulations were run with version 5.1.4 ${ }^{22}$ patched with PLUMED, version 2.3.2 ${ }^{23}$.

\subsection{System Preparation}

Initial coordinates for the complex of HLA-B*35:01 with the antigenic peptide VPLRAMTY (VY8(P5A)) were obtained from the crystal structure of HLA-B*35:01(A49P) with VPLRPMTY (VY8) (PDB ID: 1A1N). The erroneous proline residue P49 in the MHC I 
molecule and the proline at the fifth position of the antigenic peptide were mutated to alanines using the program package PyMol. The potential energy of the modified crystal structure including the crystallographic water molecules was minimized without constraints by 500 steps of steepest descent integration. During the energy minimization, no periodic boundary conditions were applied, and the non-bonded interactions were treated by the group cut-off scheme with a cut-off radius $r_{\text {cutoff }}=1 \mathrm{~nm}$ for both Coulomb and LennardJones $(12,6)$ interactions. The resulting structure was solvated in a rhombic dodecahedron simulation box such that the peptide-MHC I complex (pMHC I) and its periodic image were separated by at least a 2 nm-layer of water molecules. To obtain a neutral system with $150 \mathrm{mM} \mathrm{NaCl}, 131$ randomly selected water molecules were replaced by $70 \mathrm{Na}^{+}$and 61 $\mathrm{Cl}^{-}$ions. The potential energy of the system was minimized again by 500 steps of steepest descent integration, with non-bonded interactions treated by the Verlet cut-off scheme.

\subsection{Equilibration}

The configuration obtained from the last energy minimization was equilibrated in the NpTensemble with position restraints with a force constant $k=1000 \mathrm{kJmol}^{-1} \mathrm{~nm}^{-2}$ on the protein heavy atoms. After Maxwell-Boltzmann-distributed, random initial velocities had been generated, configurations were propagated by the Verlet leap-frog MD integrator for $20 \mathrm{~ns}$ in steps of $4 \mathrm{fs}$. They were saved to disk every 2500 steps, i.e., every 10 ps. The large time step could be employed because hydrogen atoms were replaced by virtual sites, and bonds in the protein and bonds and angles in the water molecules were restrained by $L I N C S^{24,25}$ and SETTLE ${ }^{26}$, respectively. Periodic boundary conditions were applied in all three spatial directions. Non-bonded interactions were treated by the Verlet cut-off scheme. Using Particle-Mesh-Ewald (PME) summation ${ }^{27}$, Coulomb interactions were calculated directly up

to a cut-off radius $r_{\text {cutoff }}=1.0 \mathrm{~nm}$ and evaluated in reciprocal space beyond. Lennard-Jones (LJ) $(12,6)$ interactions were truncated at a cut-off radius $r_{\text {cutoff }}=1.0 \mathrm{~nm}$ and shifted to zero at this point ${ }^{28}$. An analytical correction was applied to the energy and the pressure to 
compensate for the LJ interactions neglected beyond the cut-off. The temperature was kept at $300 \mathrm{~K}$ by two velocity-rescaling thermostats with a stochastic term and a time constant $\tau_{T}=0.1 \mathrm{ps}$, one of them coupled to the pMHC I and the other coupled to the water and the ions. The pressure was controlled by one isotropic Berendsen barostat to obtain an average pressure of 1 bar. The barostat's time constant amounted to $\tau_{p}=1.6 \mathrm{ps}$, and the compressibility of the protein-water solution was estimated to be $4.5 * 10^{-5} \mathrm{bar}^{-1}$.

The simulation parameters employed in the NpT-equilibration run were also applied in all following production simulations in the NVT ensemble, except for the following four changes:

1. In the simulations described below, the velocity Verlet MD integrator was used instead of the Verlet leap-frog integrator.

2. The temperature was controlled by one chain of 10 Nosé-Hoover thermostats coupled to the entire system with a time constant $\tau_{T}=0.5 \mathrm{ps}$.

3. To sample in the NVT-ensemble, pressure coupling was turned off.

4. No position restraints were applied. 


\subsection{Umbrella Sampling}

The configuration obtained after the NpT-equilibration was used to generate starting structures for all umbrella windows. For this purpose, the peptide N-terminus was slowly pulled out of the MHC I binding groove during a 1 ns simulation in the NVT ensemble using the parameters described in section 1.2. The targeted value of the Reaction Coordinate (RC) $r_{P 2-Y 99}$ and the resulting pull rate for each umbrella window are given in Tab. S1. In all windows, the harmonic force constant used for pulling amounted to $k=10000 \mathrm{kJmol}^{-1} \mathrm{~nm}^{-2}$. The final configurations of the pull runs were propagated in the NVT ensemble for 1 us using the same simulation parameters. Instead of pulling the peptide N-terminus, the selected value of $r_{P 2-Y 99}$ was enforced by a harmonic bias potential. The force constants used in the distinct umbrella windows are listed in Tab. S1.

After discarding the first $50 \mathrm{ps}$, the Potential of Mean Force (PMF) was calculated by Weighted Histogram Analysis (WHAM) ${ }^{29}$ using the program gmx wham ${ }^{30}$. Its error was estimated by 200 cycles of bootstrapping, during which the PMF was re-calculated for new random trajectories with properly distributed and autocorrelated configurations. 
Table S1: US parameters: targeted values of the Reaction Coordinate $(\mathrm{RC}) r_{P 2-Y 99}$, pull rates in the preparations runs, and harmonic force constants in the equilibrium production runs.

\begin{tabular}{|c|c|c|c|}
\hline Window & $\begin{array}{c}\text { Targeted } r_{P 2-Y 99} \\
{[\mathrm{~nm}]}\end{array}$ & $\begin{array}{c}\text { Pull rate } \\
{\left[\mathrm{nm} * \mathrm{ps}^{-1}\right]}\end{array}$ & $\begin{array}{l}\text { Harmonic force constants } \\
\quad\left[\mathrm{kJ} * \mathrm{~mol}^{-1} * \mathrm{~nm}^{-2}\right]\end{array}$ \\
\hline 1 & 0.665 & -0.0001 & 10000 \\
\hline 2 & 0.765 & 0.0000 & 10000 \\
\hline 3 & 0.865 & 0.0001 & 3500 \\
\hline 4 & 0.965 & 0.0002 & 4000 \\
\hline 5 & 1.065 & 0.0003 & 4500 \\
\hline 6 & 1.165 & 0.0004 & 3500 \\
\hline 7 & 1.265 & 0.0005 & 5000 \\
\hline 8 & 1.365 & 0.0006 & 3500 \\
\hline 9 & 1.465 & 0.0007 & 4000 \\
\hline 10 & 1.565 & 0.0008 & 4500 \\
\hline 11 & 1.665 & 0.0009 & 3500 \\
\hline 12 & 1.765 & 0.0010 & 4000 \\
\hline 13 & 1.865 & 0.0011 & 3500 \\
\hline 14 & 1.965 & 0.0012 & 3500 \\
\hline 15 & 2.065 & 0.0013 & 3500 \\
\hline 16 & 2.165 & 0.0014 & 3500 \\
\hline 17 & 2.265 & 0.0015 & 3500 \\
\hline 18 & 2.365 & 0.0016 & 3500 \\
\hline 19 & 2.465 & 0.0017 & 3500 \\
\hline 20 & 2.565 & 0.0018 & 3500 \\
\hline 21 & 2.665 & 0.0019 & 4500 \\
\hline 22 & 2.765 & 0.0020 & 4500 \\
\hline 23 & 2.865 & 0.0021 & 3500 \\
\hline 24 & 2.965 & 0.0022 & 3500 \\
\hline
\end{tabular}




\subsection{Bias Exchange Umbrella Sampling}

For the Bias Exchange Umbrella Sampling (BEUS) simulations, it was tried to reproduce the setup of the US simulations as closely as possible. However, to ensure sufficient overlap between the distributions of the bias potential energy at neighboring windows to observe frequent exchanges, the targeted values of the Reaction Coordinate (RC) $r_{P 2-Y 99}$ and the harmonic force constants used to enforce the selected value of $r_{P 2-Y 99}$ had to be modified (Tab. S2). Therefore, the starting structures for the BEUS simulation were obtained from the pull runs described in section 1.3 as follows: if the targeted value of $r_{P 2-Y 99}$ had not been changed in the umbrella window, the same starting structure as in the US simulation was taken; if the targeted value of $r_{P 2-Y 99}$ had been changed, the starting structure was taken from the umbrella window of the US simulation whose targeted value of $r_{P 2-Y 99}$ was closest.

These initial configurations were propagated in the NVT ensemble for $1 \mu \mathrm{s}$ employing the same simulation parameters as in section 1.3, except for the modified set of $\mathrm{RC}$ values and harmonic force constants (Tab. S2). Configurational exchanges among neighboring windows were attempted every 2 ps. With this setup, the probability for a configuration to stay in its current window ranged from $50 \%$ to $70 \%$.

After discarding the first $1 \mathrm{~ns}$ as equilibration time, the Potential of Mean Force (PMF) and its statistical error were calculated in the same way as for the US simulations described in section 1.3. 
Table S2: BEUS parameters: targeted values of the $\mathrm{RC} r_{P 2-Y 99}$ and harmonic force constants in the equilibrium production runs.

\begin{tabular}{ccc}
\hline Window & $\begin{array}{c}\text { Targeted } r_{P 2-Y 99} \\
{[\mathrm{~nm}]}\end{array}$ & $\begin{array}{c}\text { Harmonic force constants } \\
{\left[\mathrm{kJ} * \mathrm{~mol}^{-1} * \mathrm{~nm}^{-2}\right]}\end{array}$ \\
\hline 1 & 0.665 & 1000 \\
2 & 0.790 & 1000 \\
3 & 0.900 & 1000 \\
4 & 0.942 & 1300 \\
5 & 0.970 & 1500 \\
6 & 1.010 & 1500 \\
7 & 1.053 & 1200 \\
8 & 1.100 & 900 \\
9 & 1.173 & 600 \\
10 & 1.280 & 500 \\
11 & 1.372 & 500 \\
12 & 1.472 & 500 \\
13 & 1.577 & 500 \\
14 & 1.677 & 500 \\
15 & 1.770 & 500 \\
16 & 1.853 & 500 \\
17 & 1.942 & 500 \\
18 & 2.045 & 500 \\
19 & 2.145 & 500 \\
20 & 2.244 & 500 \\
21 & 2.334 & 500 \\
22 & 2.430 & 500 \\
23 & 2.530 & 500 \\
24 & 2.633 & 500 \\
\hline & &
\end{tabular}




\subsection{Replica Exchange with Solute Tempering 2}

To perform Replica Exchange with Solute Tempering 2 (REST2) simulations with distinct effective temperatures, the potential energy of a selected "heated region" has to be scaled. For the pMHC I under study, the heated region included the MHC I heavy chain residues 4-11, 23-36, 46-85, 93-101, 112-118, 122-126, and 137-180 and the complete antigenic peptide. The potential scaling was done with the program package PLUMED, version 2.3.2, i.e., all non-bonded parameters and the force constants of the dihedral angles were adjusted according to the potential scaling described in section 2.3 in the main text.

The starting structures for the REST2 simulations were generated from the final configuration of the NpT-equilibration described in section 1.2. At each effective temperature (Tab. S3), this configuration was equilibrated again in the NpT ensemble for $1 \mathrm{~ns}$ using the same simulation parameters as for the first NpT-equilibration. To avoid that replica exchanges are hampered because the simulation boxes at neighboring effective temperatures have different volumes, the box dimensions at all effective temperatures were set to the box dimensions of the final configuration of the NpT-equilibration at the lowest effective temperature.

These initial configurations were propagated two times for $2 \mu$ using the simulation parameters for NVT-production simulations described in section 1.2. Configurational exchanges among neighboring effective temperatures were attempted every $2 \mathrm{ps}$. With this setup, the probability for a configuration to stay in its current window ranged from $60 \%$ to $70 \%$.

To obtain the Potential of Mean Force (PMF), the distance $r_{P 2-Y 99}$ was calculated at the lowest effective temperature of both REST2 simulations, and the normalized histogram of the $\mathrm{RC} h\left(r_{P 2-Y 99}\right)$ was computed. Because the sampling in REST2 simulations is not biased, $h\left(r_{P 2-Y 99}\right)$ could be converted into a PMF using the relation $\Delta F\left(r_{P 2-Y 99}\right)=-k_{B} T \ln \left(h\left(r_{P 2-Y 99}\right)\right)$. The PMF of both REST2 simulations were averaged and the error of the average PMF was estimated to be the corresponding standard deviation. 
Table S3: Effective temperatures in the REST2 simulations.

\begin{tabular}{cc}
\hline Replica index & Effective temperature $[\mathrm{K}]$ \\
\hline 1 & 300.0 \\
2 & 307.8 \\
3 & 315.8 \\
4 & 324.0 \\
5 & 332.4 \\
6 & 341.1 \\
7 & 350.0 \\
8 & 359.1 \\
9 & 368.4 \\
10 & 378.0 \\
11 & 387.8 \\
12 & 397.9 \\
13 & 408.2 \\
14 & 418.9 \\
15 & 429.7 \\
16 & 440.9 \\
17 & 452.4 \\
18 & 464.2 \\
19 & 476.2 \\
20 & 488.6 \\
\hline
\end{tabular}




\subsection{Well-Tempered Metadynamics}

For the well-tempered Metadynamics (MTD) simulations, the same NVT simulation parameters and the same set of starting configurations as for the BEUS simulations were employed. In one set of 21 well-tempered MTD simulations, which used the starting configurations of all BEUS windows but window 5, 9, and 24, the distinct simulation walkers built up their individual bias potentials, which were not communicated among the walkers. These simulations will be referred to as the MTD simulations with individual bias potentials. Each walker was run for $100 \mathrm{~ns}$. In five sets of 23 simulation walkers starting from the initial configurations of the BEUS windows 1 to 23, the history-dependent bias potentials of all walkers were instantly summed up, and all walkers used the same summed potential to bias the propagation of their configuration in phase space. These five sets will be referred to as MTD runs with pooled bias potential. In three sets (run 1, 2, and 4), the individual walkers were propagated for $100 \mathrm{~ns}$; in the remaining two sets, the simulations were stopped after 75 ns.

Apart from the different treatment of the bias potential, the MTD-specific parameters were kept mostly identical for both types of MTD simulations. In both types, the sampling of the two distances $r_{P 2-Y 99}$, the $\mathrm{RC}$, and $r_{R 62-W 167}$, which reflects the breathing motions of the MHC I binding groove, was biased. To this end, a Gaussian function with a width of $\sigma_{1}=0.1 \mathrm{~nm}$ and $\sigma_{2}=0.04 \mathrm{~nm}$ along $r_{P 2-Y 99}$ and $r_{R 62-W 167}$, respectively, was added to the bias potential in each walker every $10 \mathrm{ps}$. The initial amplitude of this Gaussian function was set to $1.0 \mathrm{kJmol}^{-1}$ and $0.8 \mathrm{kJmol}^{-1}$ for the MTD simulations with individual bias potentials and the MTD runs with pooled bias potential, respectively. For both types, the bias factor amounted to 10 , i.e., with a system temperature $T=300 \mathrm{~K}$, the temperature difference was set to $\Delta T=2700 \mathrm{~K}$. 


\subsection{Analysis Protocols}

\subsubsection{Umbrella Sampling}

For the Umbrella Sampling (US) simulation, the Potential of Mean Force (PMF) was calculated six times: after sampling $1 \mathrm{~ns}, 10 \mathrm{~ns}, 50 \mathrm{~ns}, 100 \mathrm{~ns}, 500 \mathrm{~ns}$, and $1 \mu \mathrm{s}$ in each umbrella window. Because the PMF was computed with gmx wham, the respective bootstrapping error and the Integrated Auto-Correlation Times (IACT) were obtained from the same calculation.

In addition, a Principal Component Analysis (PCA) was performed for each umbrella window using gmx covar, and the trajectory was projected onto the first PCA eigenvector using gmx anaeig.

\subsubsection{RE-Based Simulations}

The histograms of the Reaction Coordinate (RC) $r_{P 2-Y 99}$ that are shown for the three REbased simulations, the Bias Exchange Umbrella Sampling (BEUS) simulation and the two Replica Exchange with Solute Tempering 2 (REST2) simulations, were obtained during PMF calculation (Fig. 6).

To generate the 3D histograms of the relative orientations of the peptide N-terminus with respect to Y99 (Fig. $7 \&$ S3-S5), the x-, y-, and z-component of $r_{P 2-Y 99}$ were calculated. The 3D histogram $h_{\text {orient }}$ of these vectors was computed using numpy. The entries of the histogram were converted into "orientation free energies" according to $\Delta F_{\text {orient }}=-k_{B} T \ln \left(h_{\text {orient }}\right)$.

The volume of the binding groove was estimated on the basis of the positions of the $\mathrm{C}_{\alpha^{-}}$ atoms of its residues (Fig. 8a-c). For each configuration, a PCA was performed and the three first eigenvectors were aligned with the axes of the coordinate system. Because the binding groove is always longer than wide and wider than deep, the PCA enforced a standard orientation. Next, it was tried to place small cubes with volume $V=0.001 \mathrm{~nm}^{3}$ inside the binding groove. A new cube was accepted if it did not collide with any of the $\mathrm{C}_{\alpha}$-atoms, and 
if it was inside one of the ellipsoids used to describe the shape of the binding groove. The volume of the binding groove was calculated as the sum of the volumes of all cubes placed inside the binding groove. The pseudocode of the corresponding $\mathrm{C}++$ program is given in section 4.1 .

The Schlitter entropies ${ }^{31}$ of the MHC I binding groove, the antigenic peptide, and the binding groove together with the peptide were computed using gmx covar for the PCA and gmx anaeig for the actual entropy calculation (Fig. 8d \& S6). The Quasiharmonic Approximation

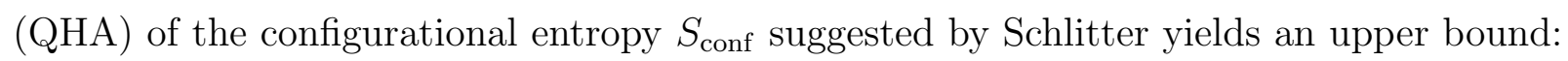

$$
S_{\text {conf }}<0.5 k_{B} \ln \left[\operatorname{det}\left(\mathbf{1}+k_{B} T e^{2} \hbar^{-2} \mathbf{M}^{1 / 2} \mathbf{C M}^{1 / 2}\right)\right]
$$

where $k_{B}$ denotes Boltzmann's constant, $T$ the temperature, $e$ Euler's number, and $\hbar$ the reduced Planck constant. The matrices $\mathbf{M}$ and $\mathbf{C}$ are the diagonal matrix of the particle masses and the covariance matrix of the particle positions, respectively. Here, $\mathbf{C}$ was defined by the vector $\vec{x}$ containing the positions of all $\mathrm{C}_{\alpha}$-atoms in the $\mathrm{MHC} \mathrm{I}$ binding groove (Tab. S4) and/or the antigenic peptide as:

$$
\mathbf{C}=\left\langle(\vec{x}-\langle\vec{x}\rangle)(\vec{x}-\langle\vec{x}\rangle)^{T}\right\rangle
$$

To study the mechanism underlying the insufficient sampling of the partially dissociated state in the two REST2 simulations (Fig. $9 \&$ S7), the distance $r_{P 2-Y 99}$ was calculated and the corresponding histogram $h\left(r_{P 2-Y 99}\right)$ was computed at all effective temperatures. For the analysis of the Metropolis Monte Carlo (MC) sampler and the MD integrator, the fully bound state was defined as $0.00 \mathrm{~nm} \leq r_{P 2-Y 99} \leq 1.05 \mathrm{~nm}$, and the partially dissociated state was given by $r_{P 2-Y 99}>1.05 \mathrm{~nm}$, accordingly. The replica exchanges mediated by the MC sampler were thus categorized as exchanges of configurations belonging to the same state and mixed exchanges. Among the mixed exchanges, exchanges in which a configuration of the fully bound state moves from the lower to the higher temperature were distinguished 
from exchanges in which a configuration from the partially bound state moves to the higher temperature. For all three types of exchanges, the probability for the exchange to be accepted was calculated as the number of successful exchanges of that kind divided by the total number of exchanges of that kind that were attempted during the REST2 simulation. Similarly, to calculate the probability for transitions from the fully bound to the partially dissociated state and vice versa during MD integration, subsequent configurations were assigned to one of the two states, and the respective probability was computed as the number of successful transitions divided by the total number of attempted transitions. However, to correctly describe the behavior of the MD integrator, only those pairs of subsequent configurations in between which no successful replica exchange took place were considered to be attempted transitions. These three MC acceptance probabilities and two MD transition probabilities were calculated at all effective temperatures for both REST2 simulations. 


\section{Free (Unbiased) MD Simulations of HLA-B*35:01 in Complex with VY8(P5A)}

a)

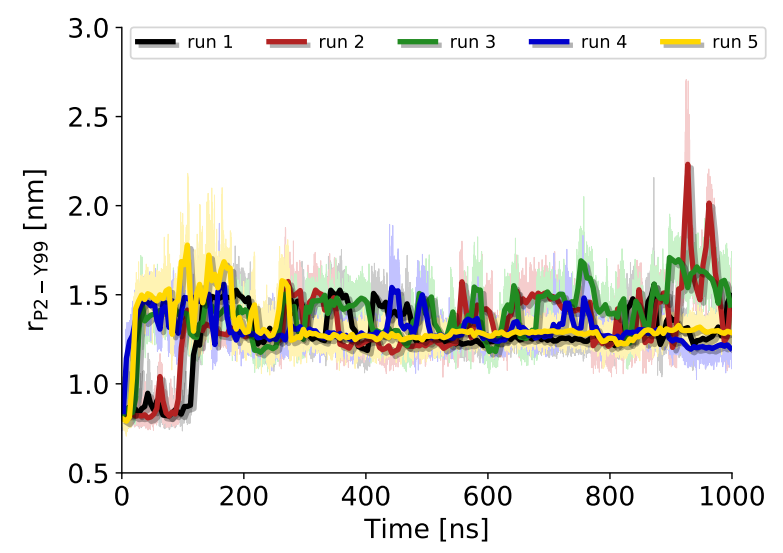

c)

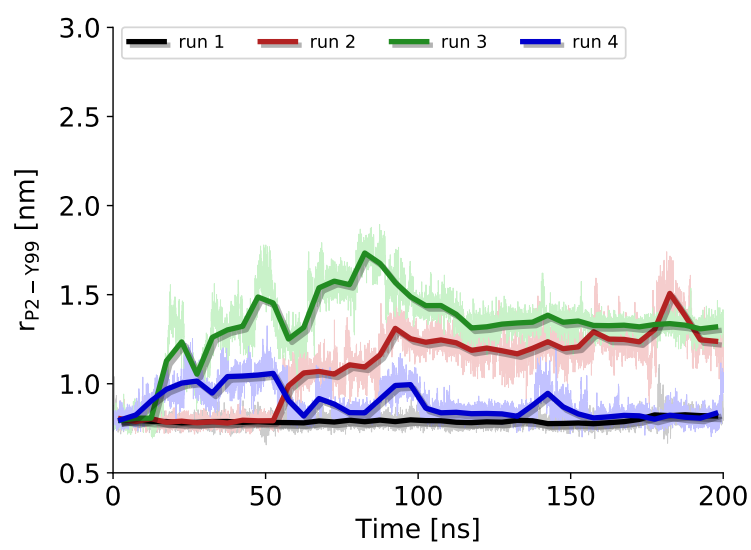

b)

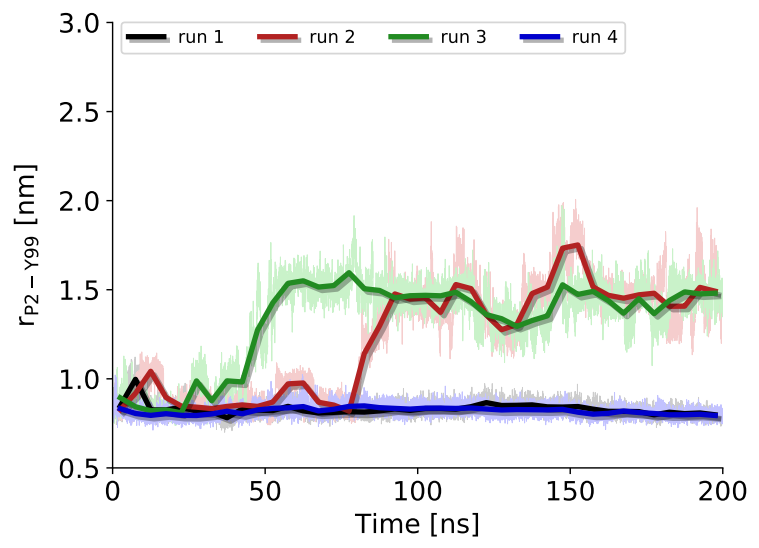

d)

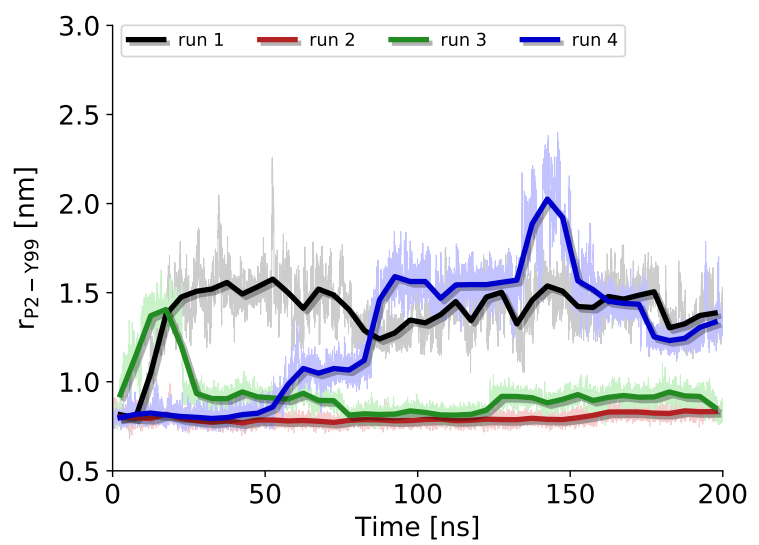

Figure S1: Dissociation of the peptide N-terminus from the MHC I binding groove in free (unbiased) MD simulations. The center-of-mass distance $r_{P 2-Y 99}$ between P2 at the peptide Nterminus and its binding partner Y99 in the binding groove is shown as a function of simulated time. The actual time series (pale shades) is overlaid by a $5 \mathrm{~ns}$ moving average (bold solid lines). Four different combinations protein force field/water model were tested: a) five $1 \mu \mathrm{s}$ simulations with AMBER99SB*-ILDNP/TIP4P, b)-d) four $200 \mathrm{~ns}$ control simulations each with AMBER99SB*-ILDNP/TIP3P, CHARMM36/TIP4P, and CHARMM36/CHARMMTIP3P, respectively. All simulations were started in the fully bound state. 


\section{A Second RC for Well-Tempered MTD}

a)

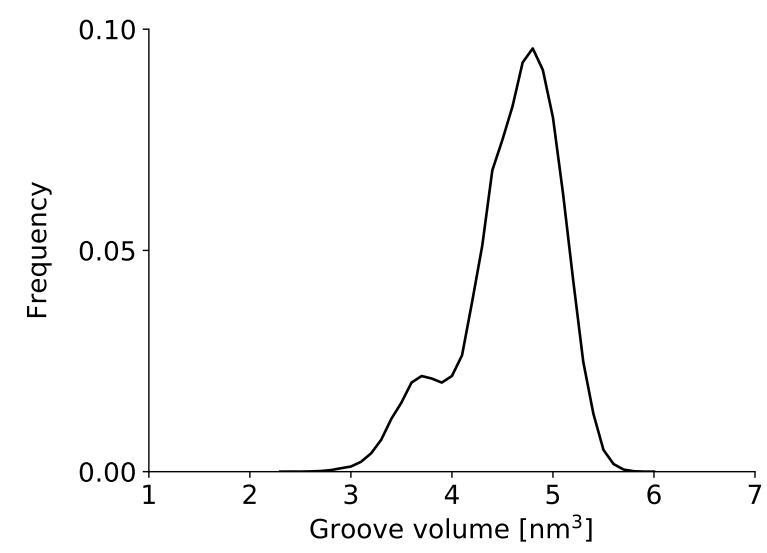

b)

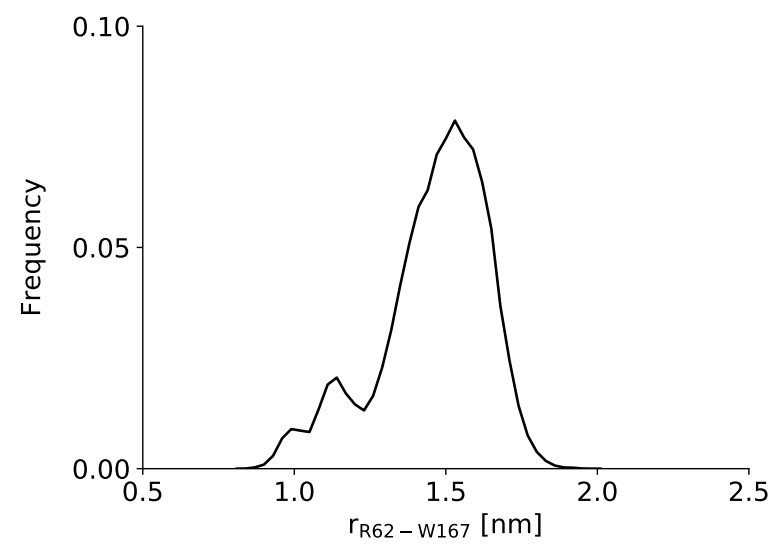

Figure $\mathrm{S} 2$ : The distance between the $\mathrm{C}_{\alpha}$-atoms of $\mathrm{R} 62$ and $\mathrm{W} 167, r_{R 62-W 167}$, is representative of the binding groove volume. Data taken from the window with $r_{P 2-Y 99}=2.633 \mathrm{~nm}$ of the BEUS simulation. 


\section{Supplementary Results of the Analysis of the RE- Based and Well-Tempered MTD Simulations}

Due to the large amount of data generated by the BEUS and the two REST2 simulations, the sampling of the configurational entropy of the antigenic peptide is discussed on the basis of only four example histograms in the main text (Fig. 7). Here, the complete set of the 3D histograms at all values of the $\mathrm{RC}$ simulated is presented for all three simulations (Fig. S3-S5). Moreover, the Schlitter entropy was not only calculated for the MHC I binding groove (Fig. 8), but also for the antigenic peptide and together for the binding groove and the antigenic peptide (Fig. S6). Furthermore, the mechanism due to which the REST2 simulations fail to sufficiently sample the partially dissociated state of the pMHC I under study (Fig. 9) could be reproduced in the second REST2 simulation (Fig. S7). The 2D well-tempered MTD simulations were performed with and without pooling the bias potential of the distinct simulation walkers. The PMF obtained from a set of 21 simulations in which each walker only senses the individual bias potential that it has accumulated itself is shown in Fig. S8 and can be compared with the PMF from the simulations with pooled bias potential shown in the main text (Fig. 10). Moreover, the time evolution of the RC in the individual walkers of run 2 with pooled bias potential is provided in Fig. S9.

Last, this section contains the pseudocode of the $\mathrm{C}++$ script used to calculate the volume of the binding groove. 

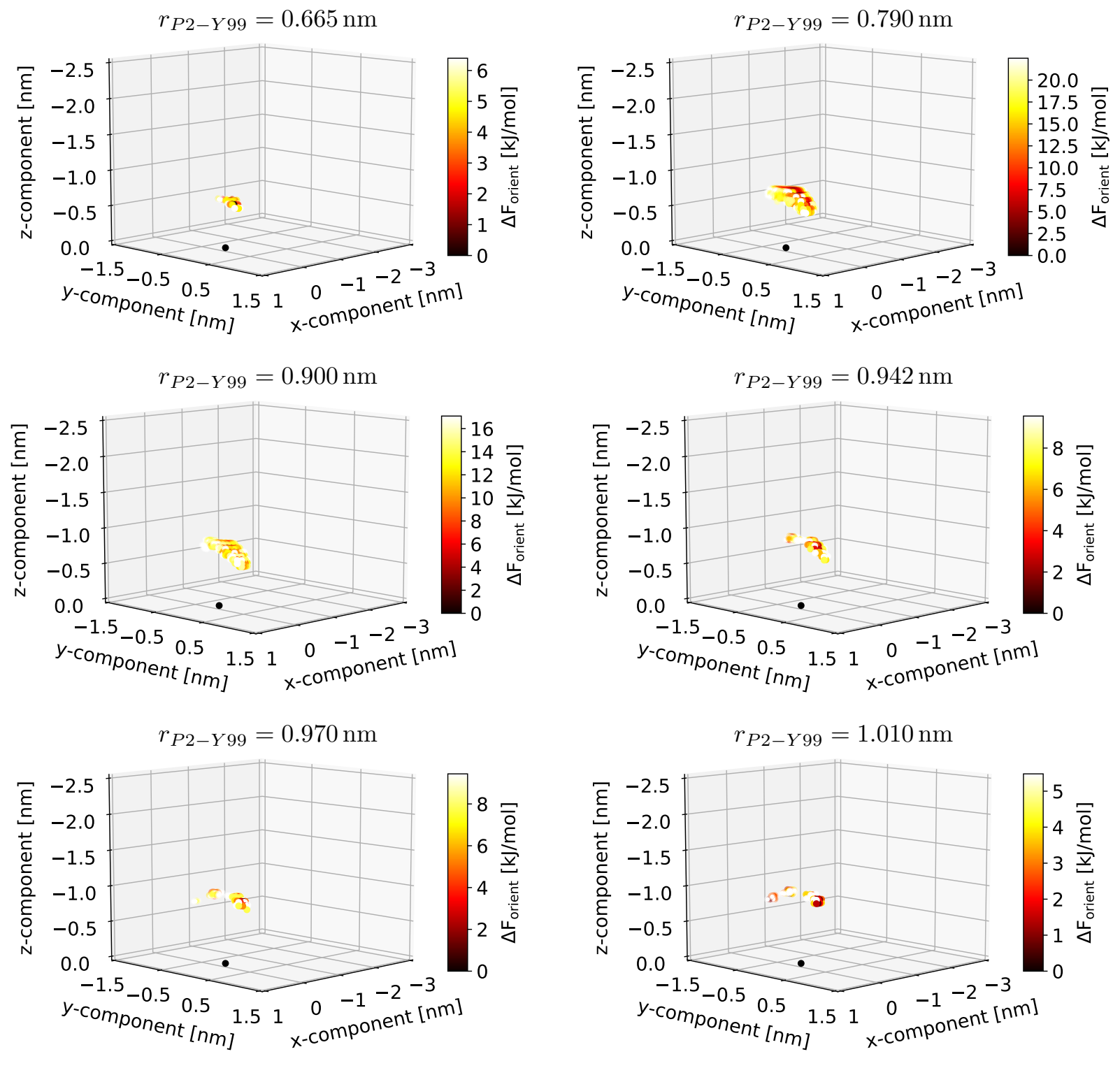

$r_{P 2-Y 99}=1.053 \mathrm{~nm}$
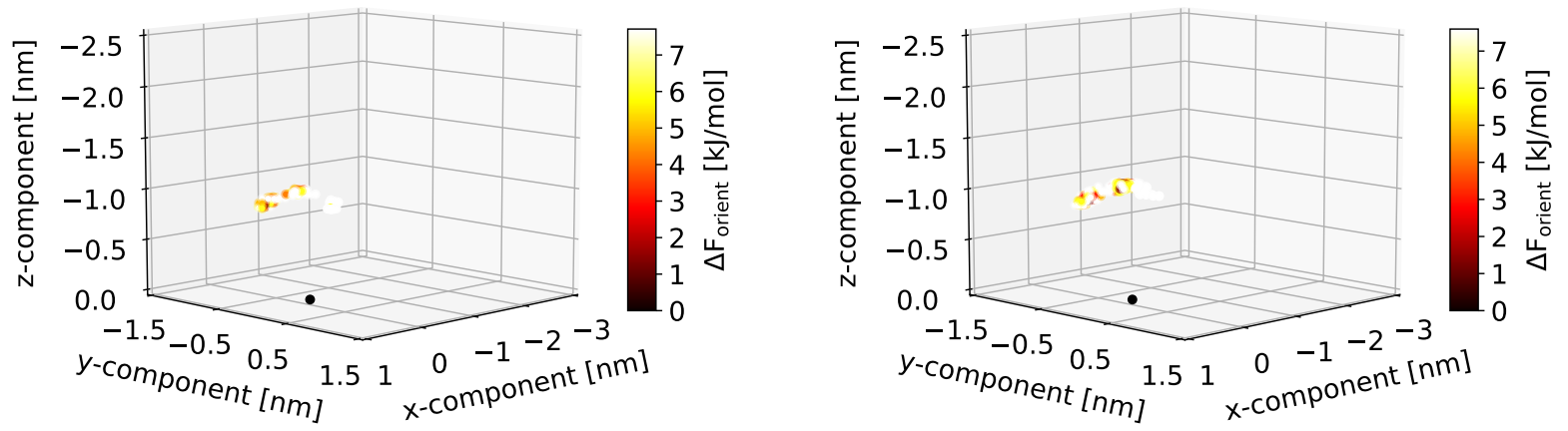

Figure S3: 3D histograms of the relative orientations of the peptide N-terminus with respect to Y99 at distinct values of the RC $r_{P 2-Y 99}$, using the free energy $\Delta F_{\text {orient }}$ defined in Fig. 7. 1 bin $\triangleq 0.05 \mathrm{~nm}$ and the black dot marks the center of mass of Y99. Data were taken from REST2 simulation 1. 
$r_{P 2-Y 99}=1.173 \mathrm{~nm}$

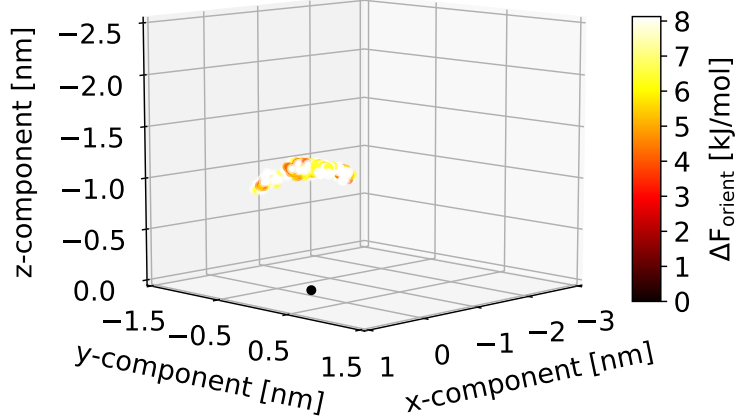

$r_{P 2-Y 99}=1.372 \mathrm{~nm}$

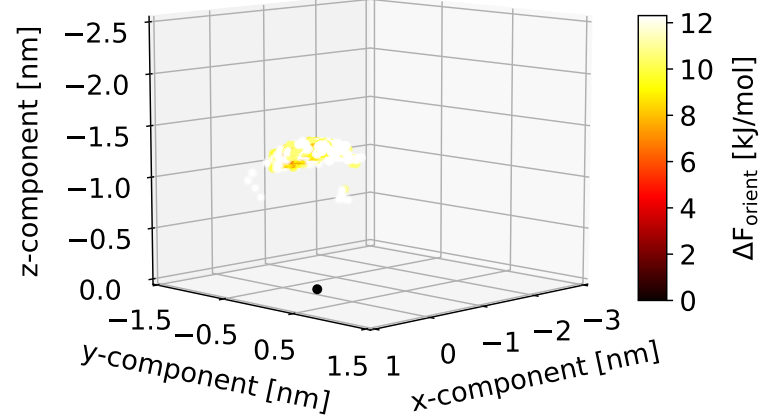

$r_{P 2-Y 99}=1.577 \mathrm{~nm}$

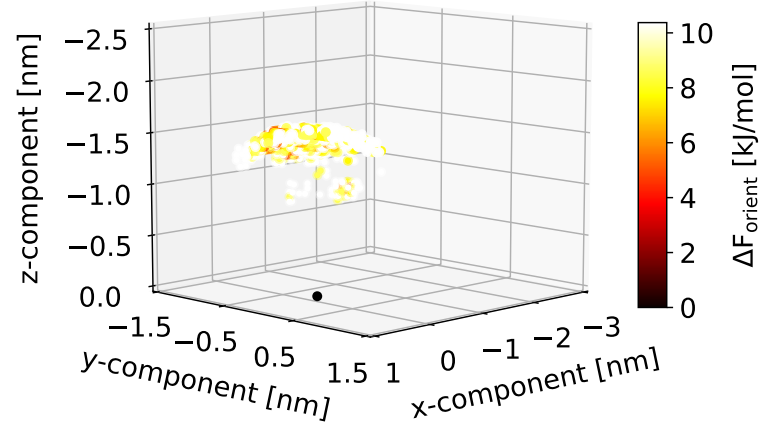

$r_{P 2-Y 99}=1.770 \mathrm{~nm}$

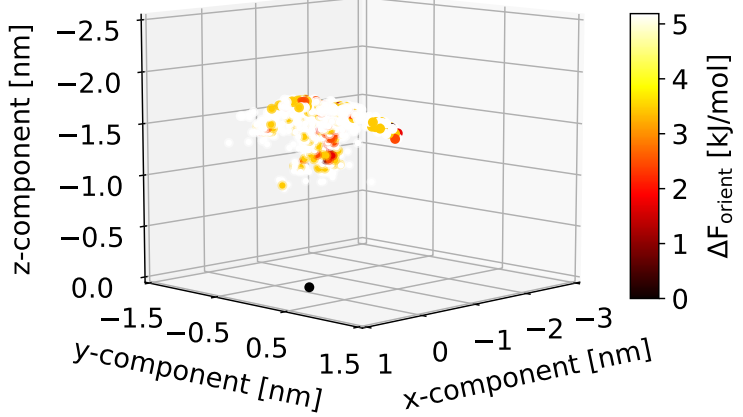

$r_{P 2-Y 99}=1.280 \mathrm{~nm}$

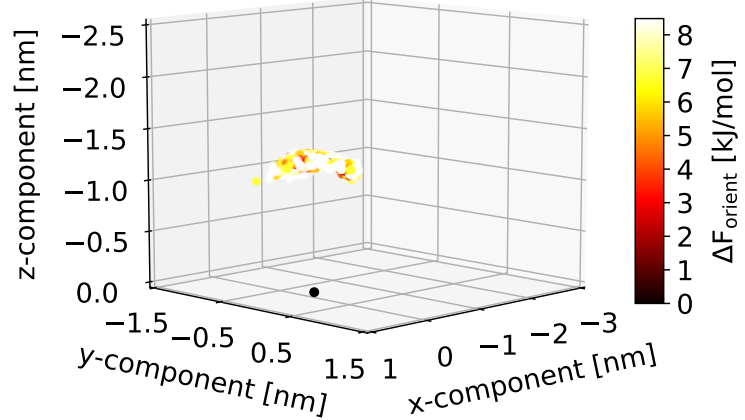

$r_{P 2-Y 99}=1.472 \mathrm{~nm}$

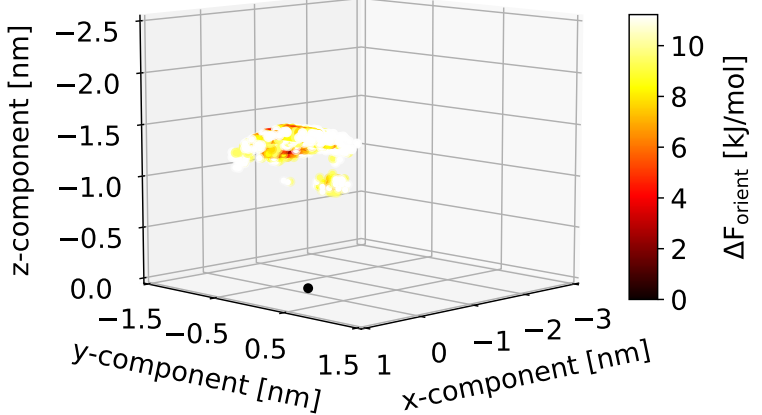

$r_{P 2-Y 99}=1.677 \mathrm{~nm}$

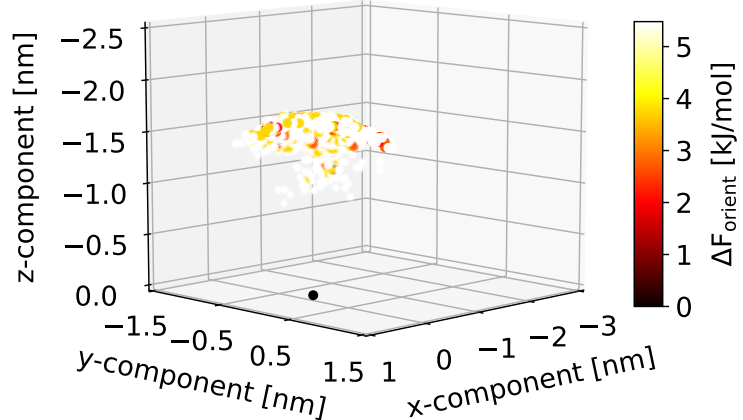

$r_{P 2-Y 99}=1.853 \mathrm{~nm}$

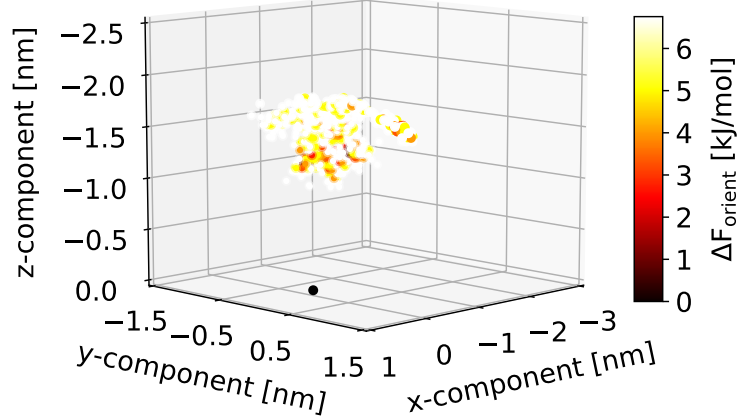

Figure S3: Continued. 

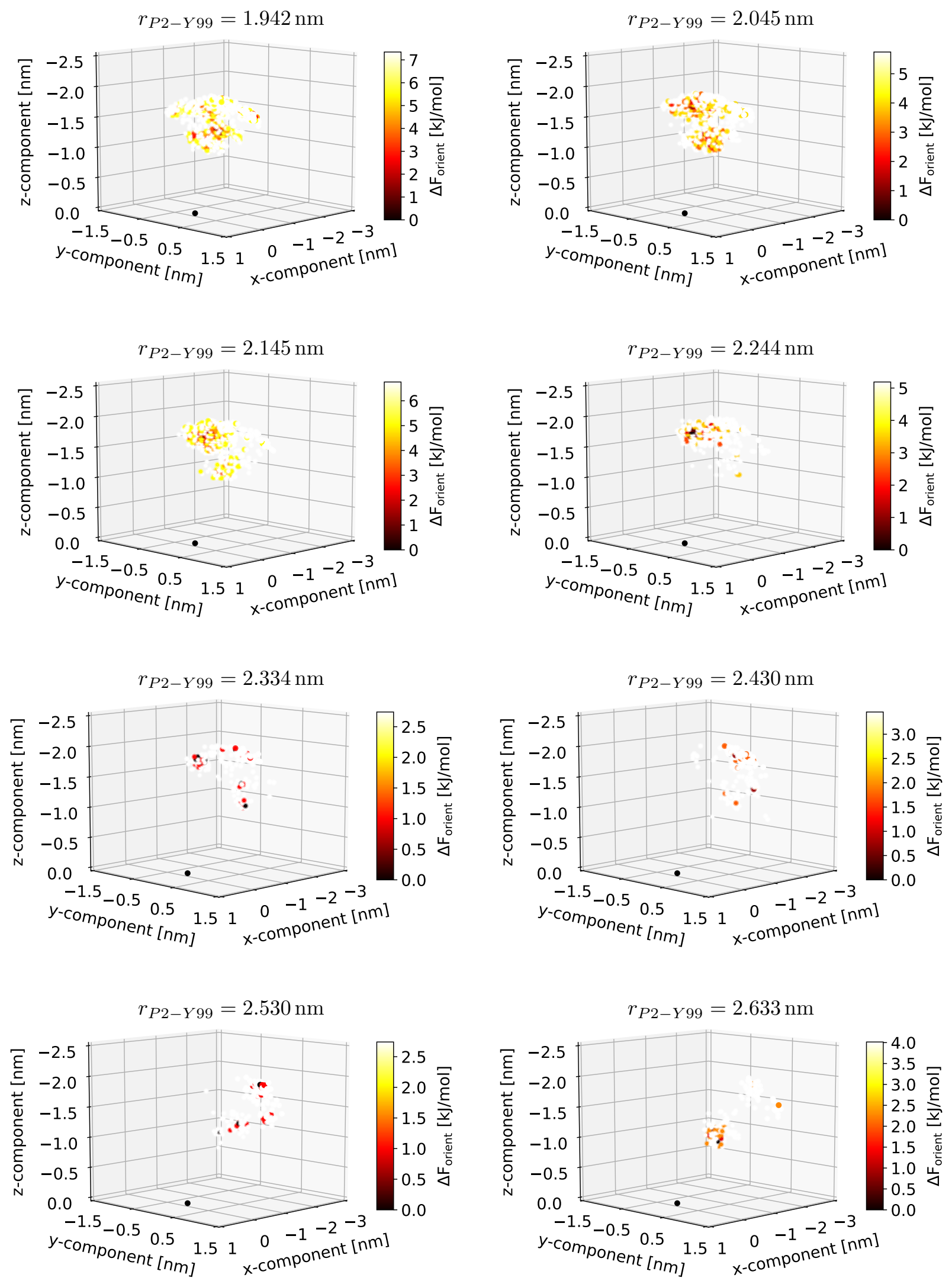

Figure S3: Continued. 

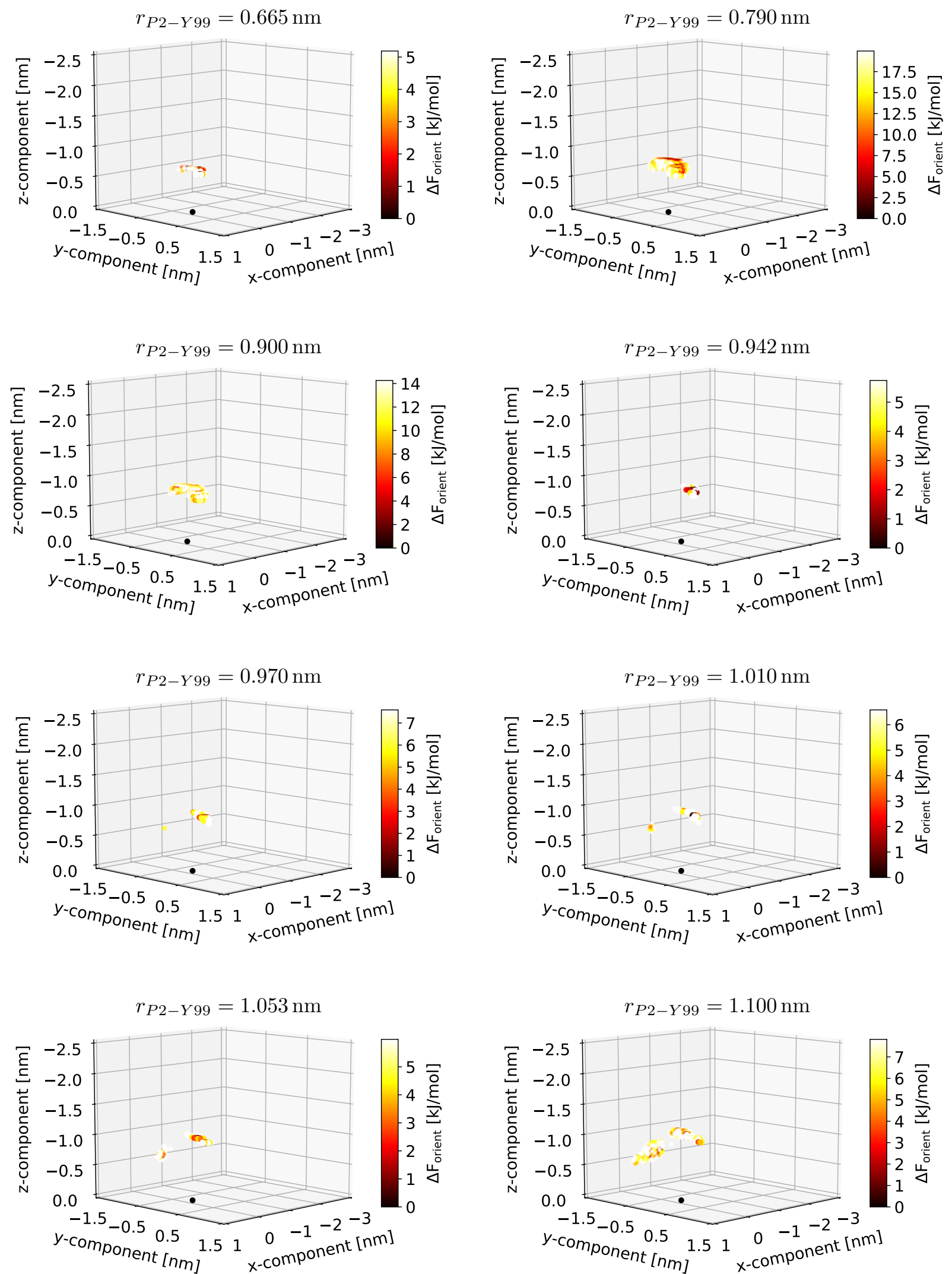

Figure S4: Same as Fig. S3, but data were taken from REST2 simulation 2. 

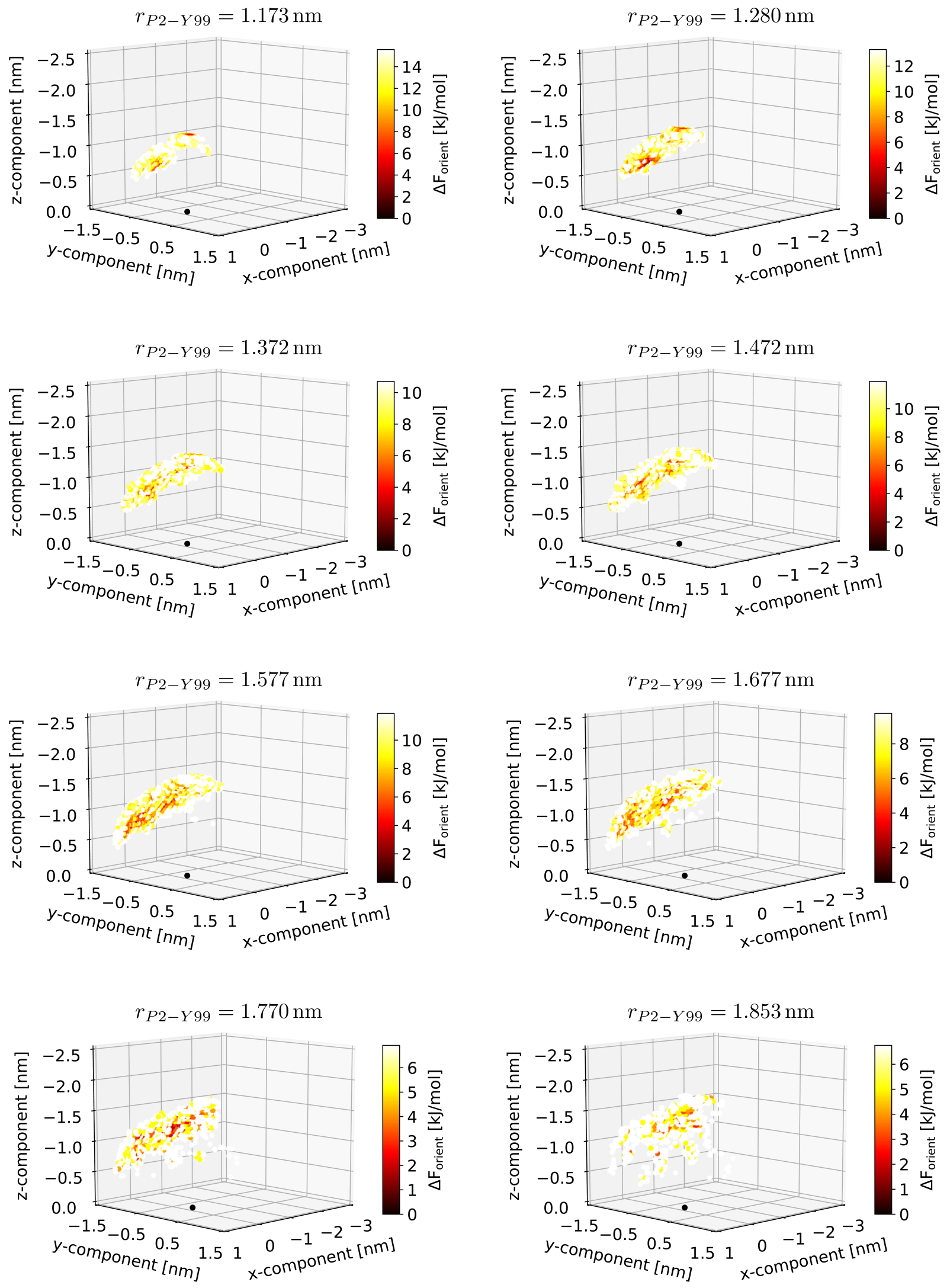

Figure S4: Continued. 

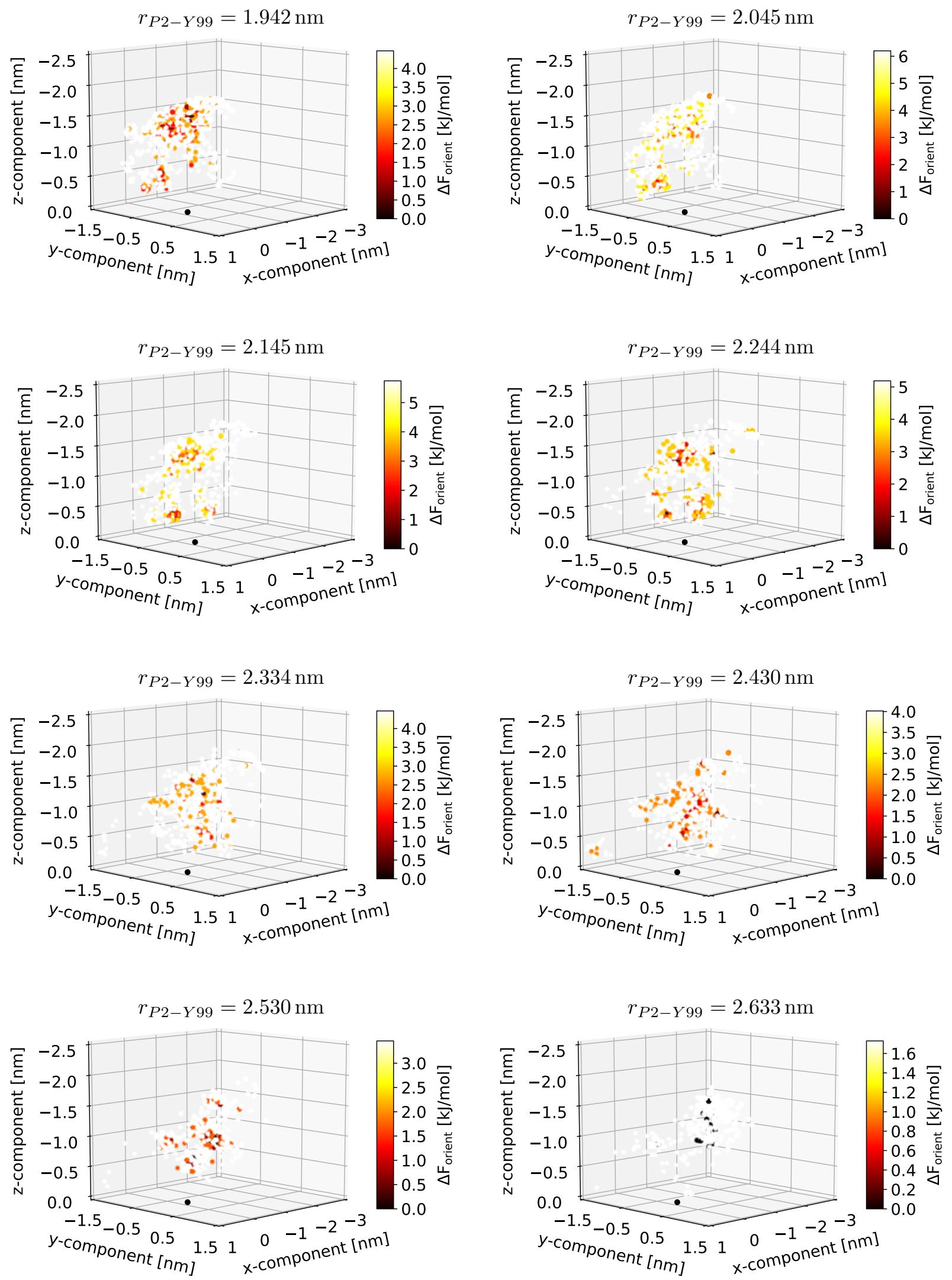

Figure S4: Continued. 

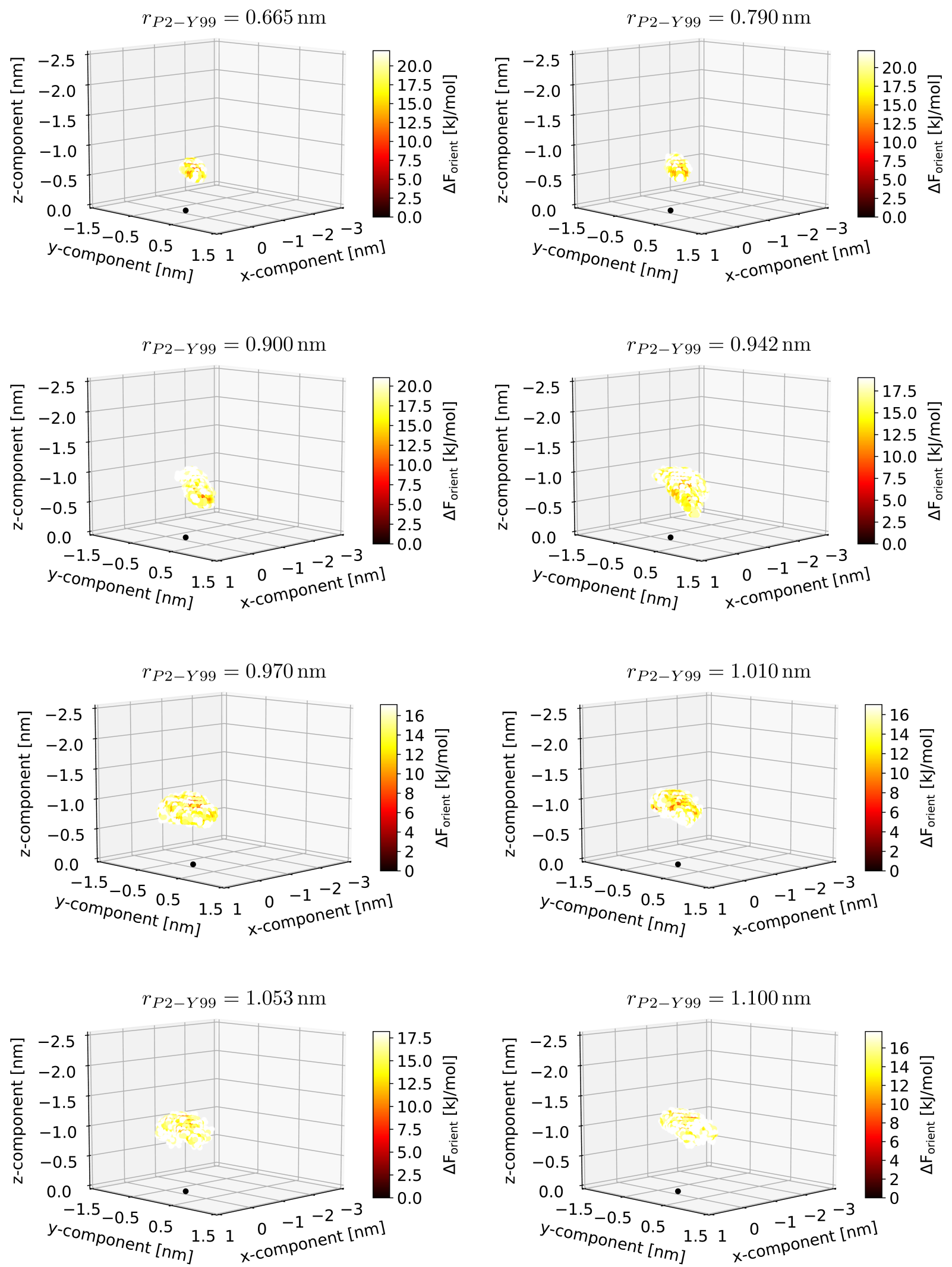

Figure S5: Same as Fig. S3, but data were taken from the BEUS simulation. 

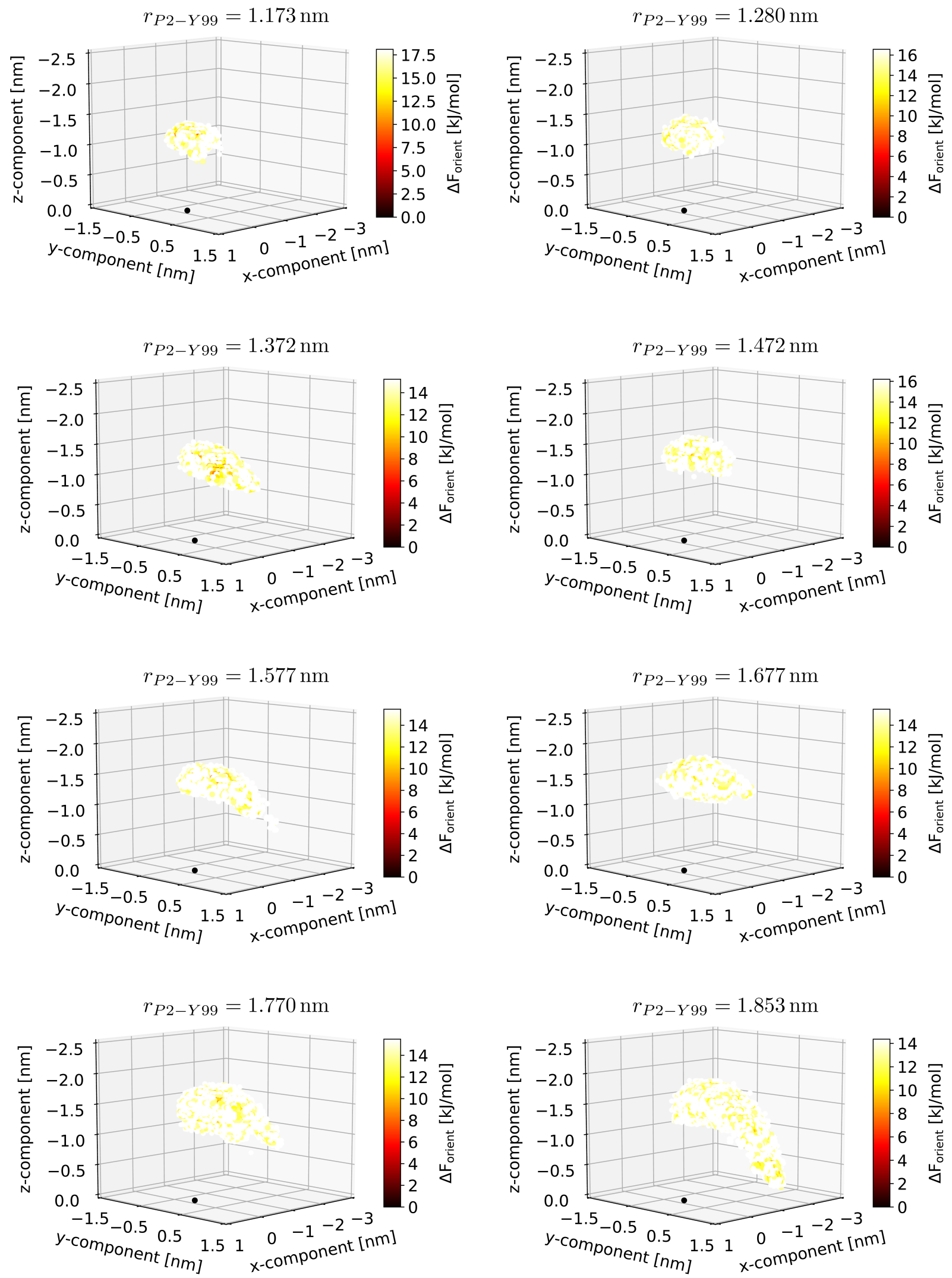

Figure S5: Continued. 

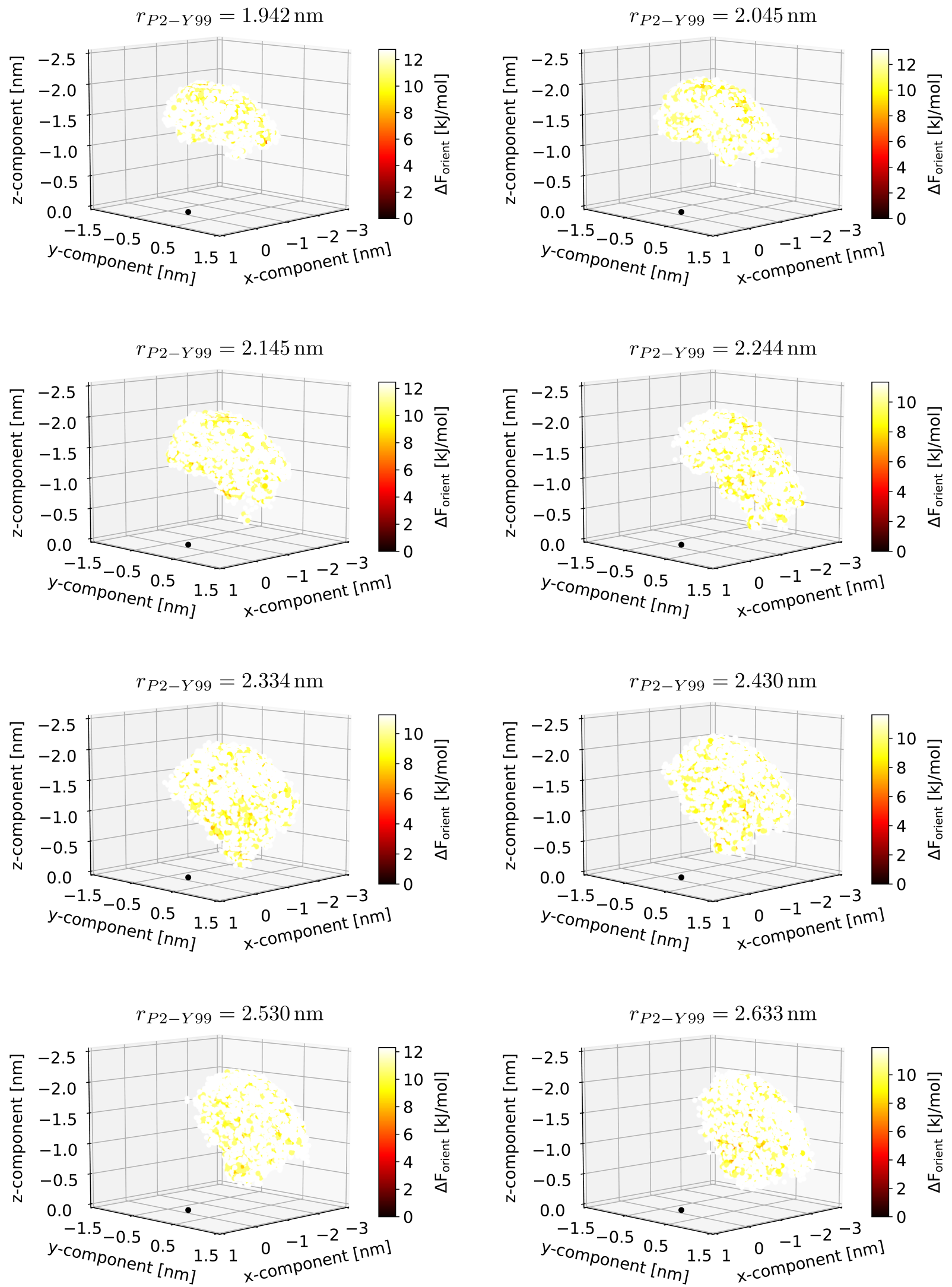

Figure S5: Continued. 
a)

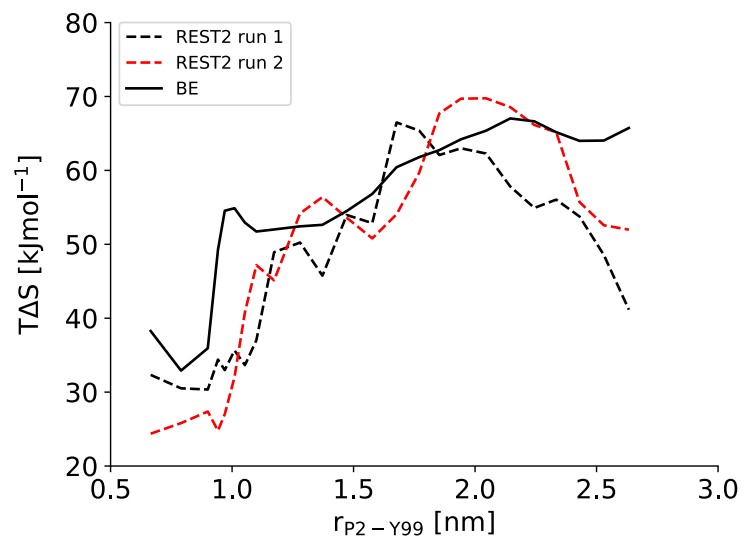

b)

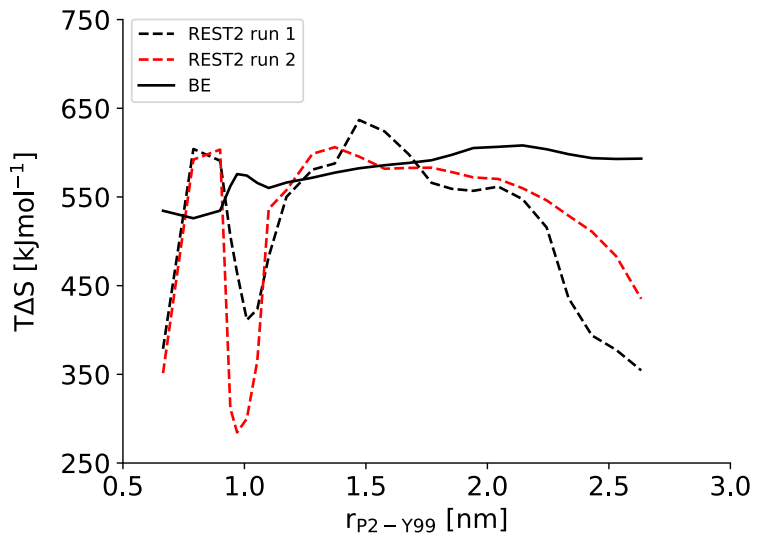

Figure S6: Schlitter entropies of: a) the antigenic peptide, b) the binding groove and the antigenic peptide (calculated at once). The Schlitter entropies of the binding groove and the antigenic peptide are not exactly additive due to correlations between the two. Therefore, the entropy shown in $b$ is not the sum of the individual entropy of the binding groove (shown in Fig. 8) and the entropy of the antigenic peptide (shown in a). 
a)

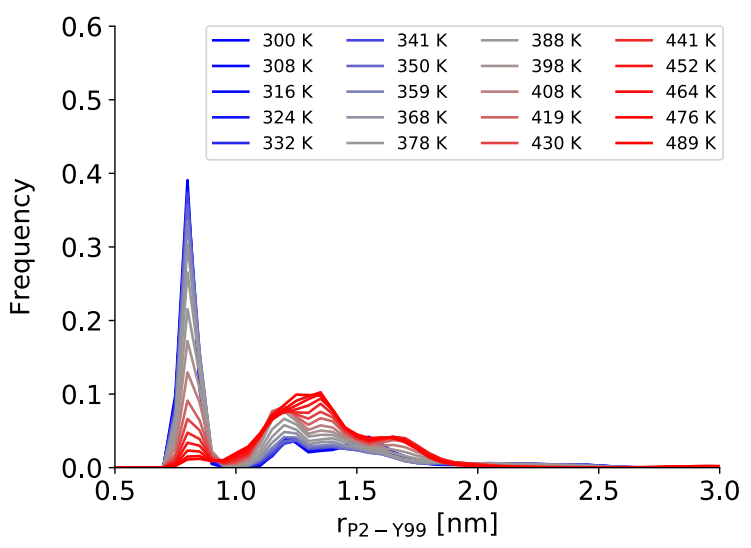

c)

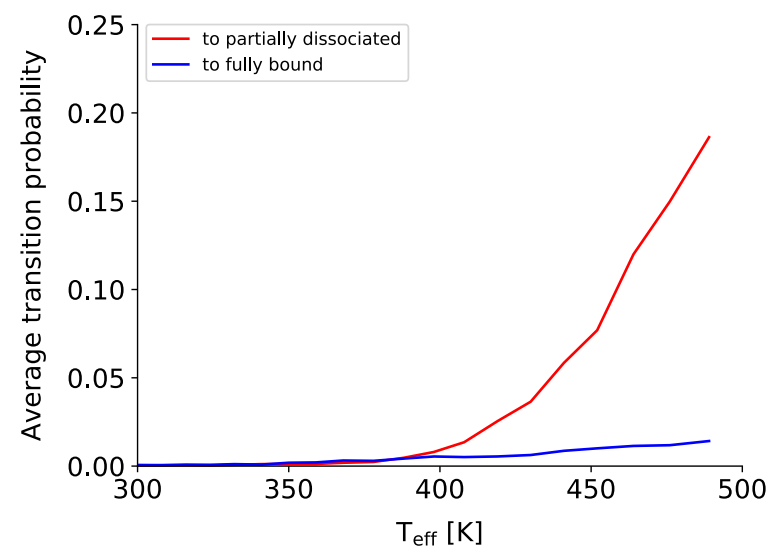

b)

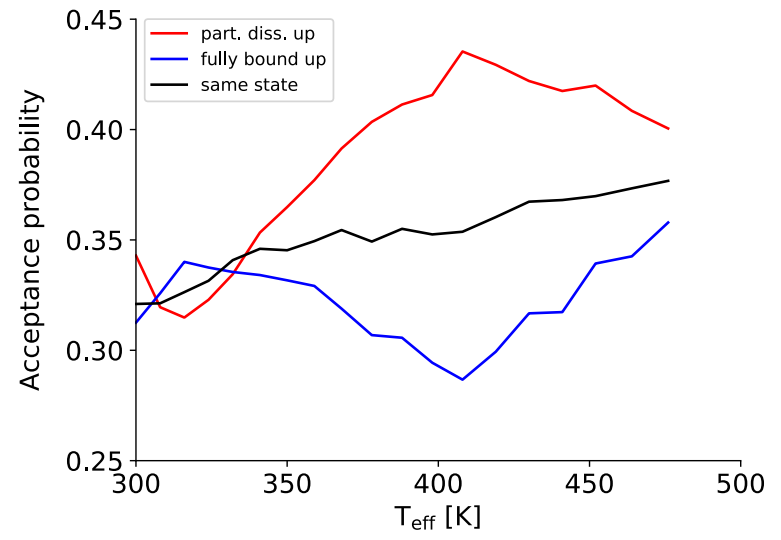

d)

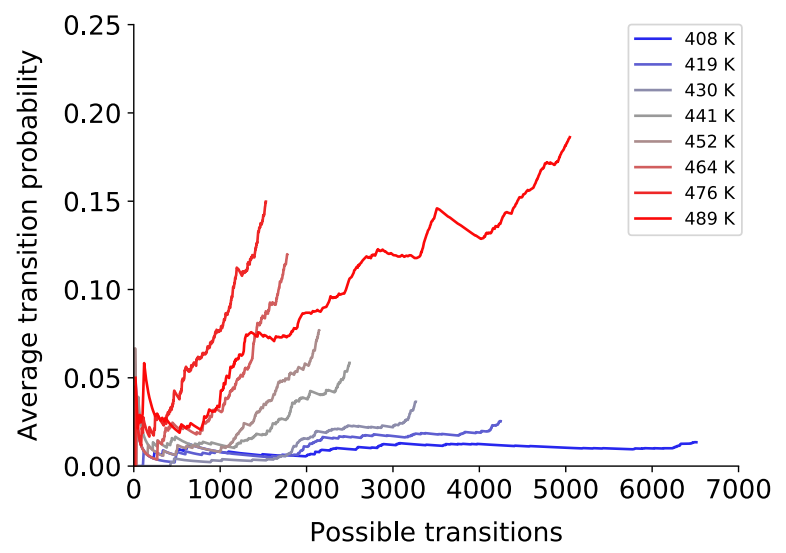

Figure S7: Same analysis as presented in Fig. 9, but data were taken from REST2 simulation 2 . 


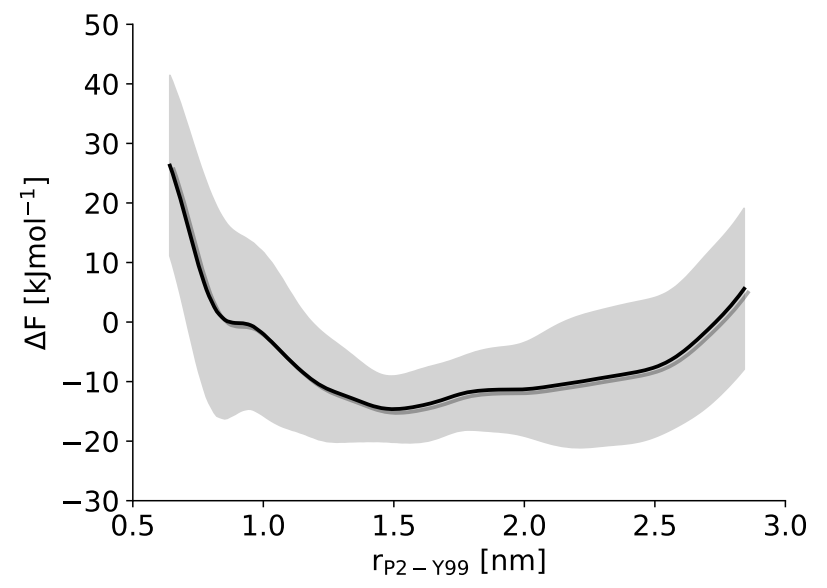

Figure S8: Average PMF with standard deviation obtained from 21 well-tempered MTD simulations with individual bias potentials. 
1)

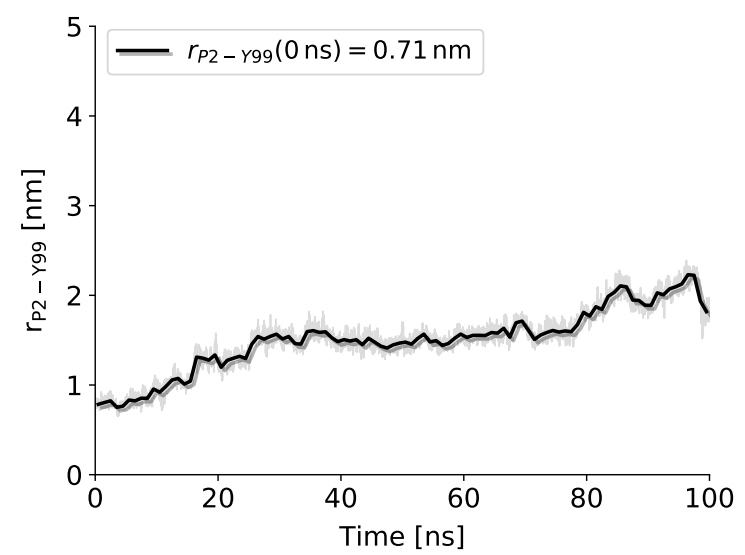

3)

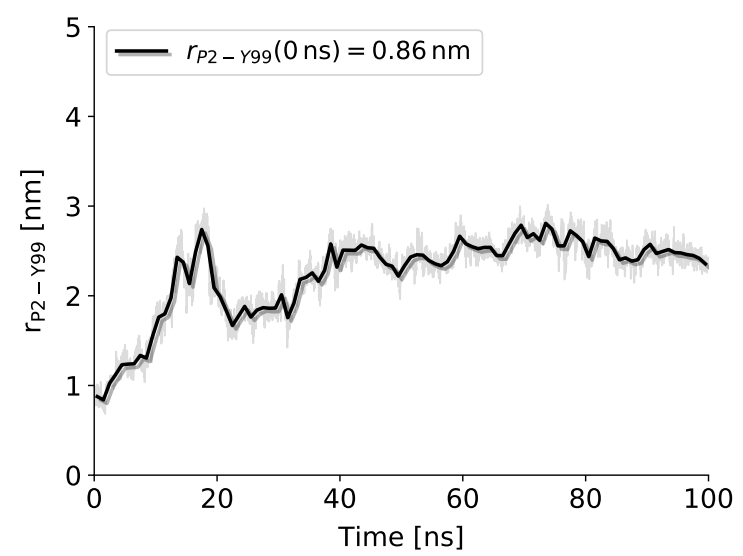

5)

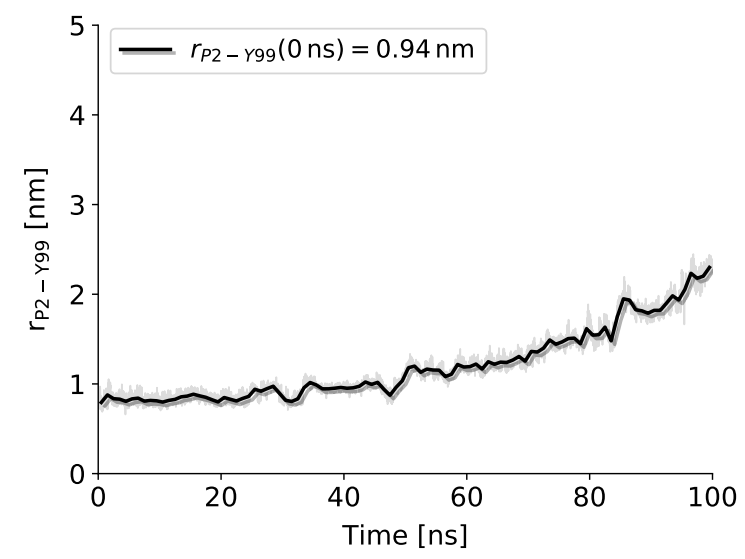

2)

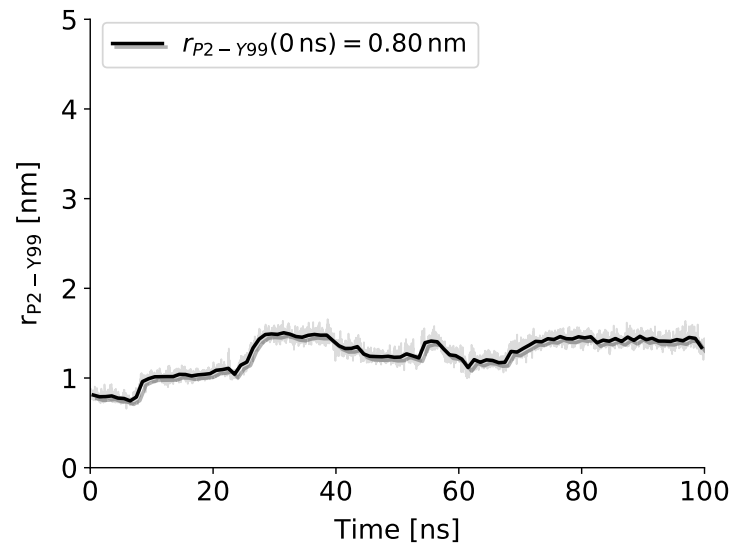

4)

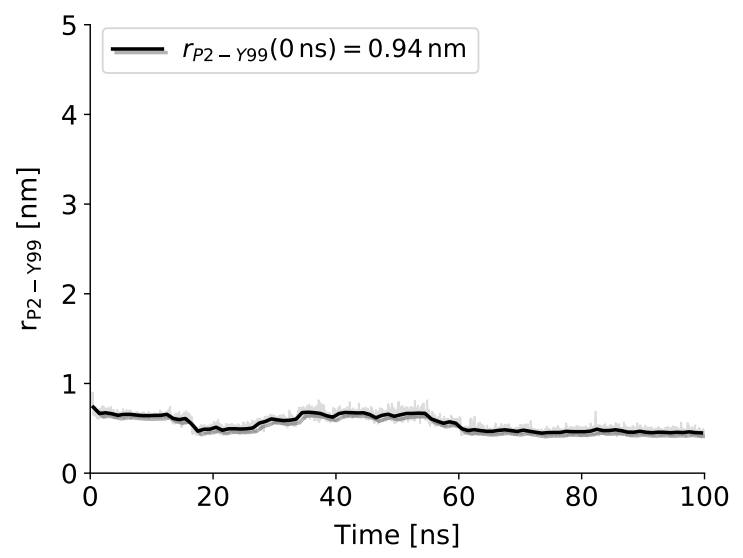

6)

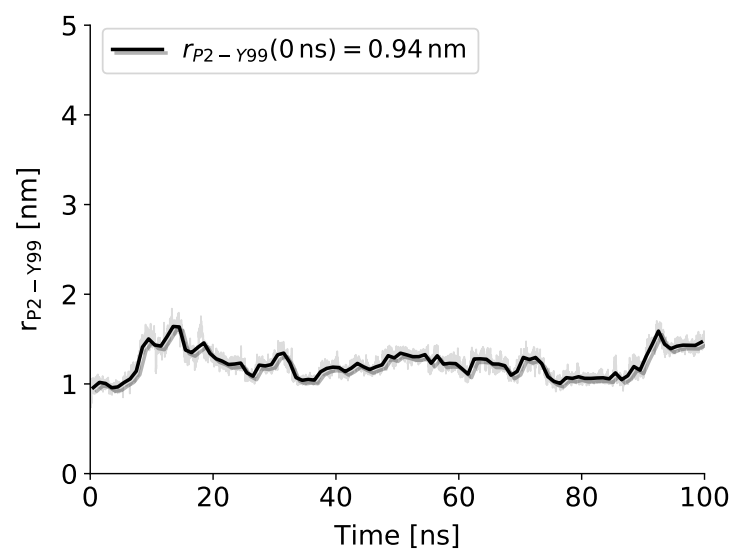

Figure S9: Time evolution with 1 ns moving average of $r_{P 2-Y 99}$ in single simulation walkers taken from run 2 as in Fig. 10. 
7)

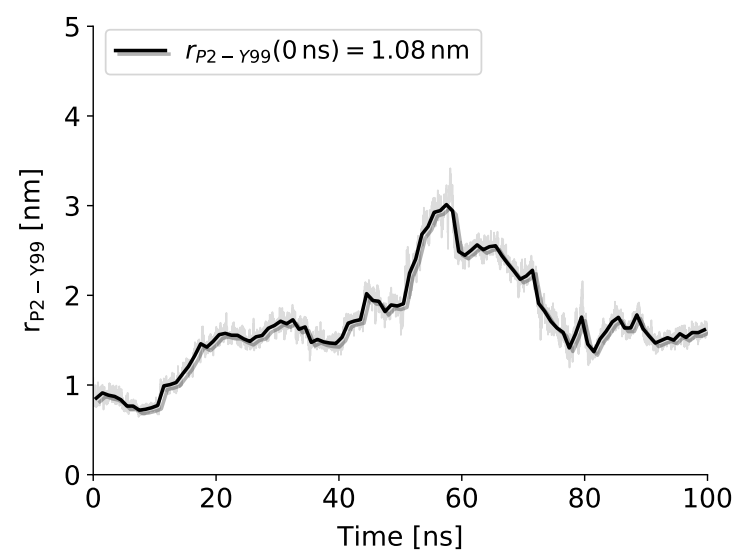

9)

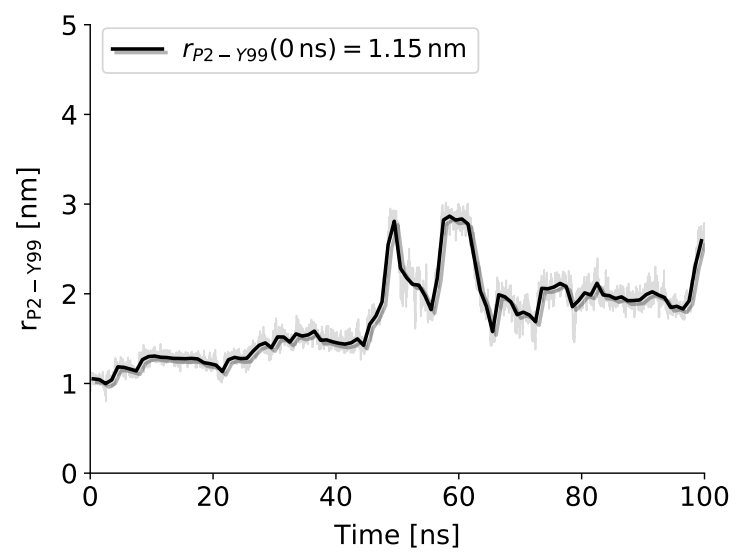

11)

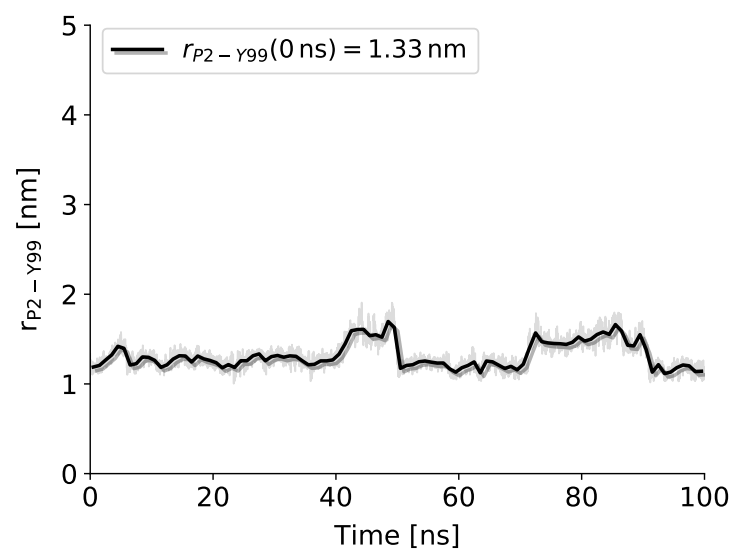

8)

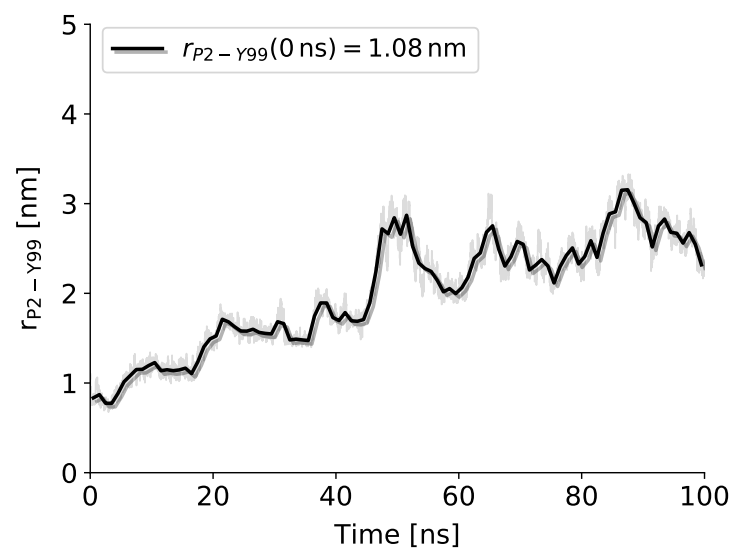

10)

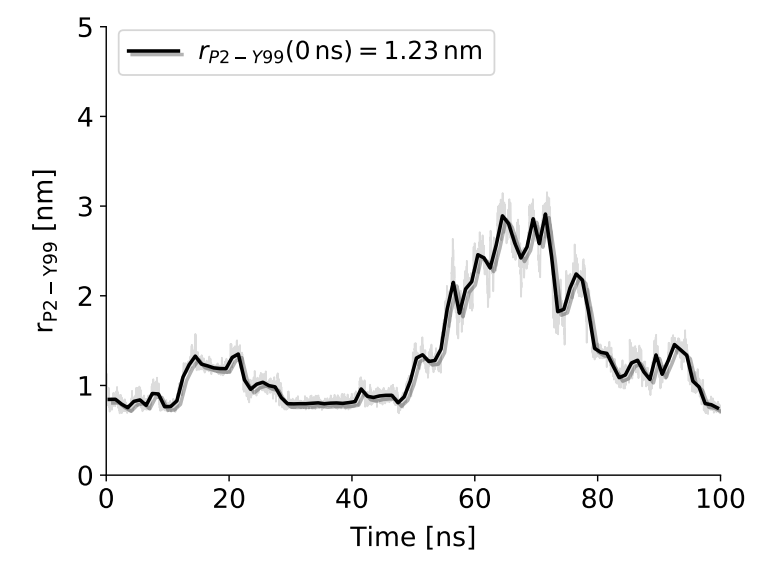

12)

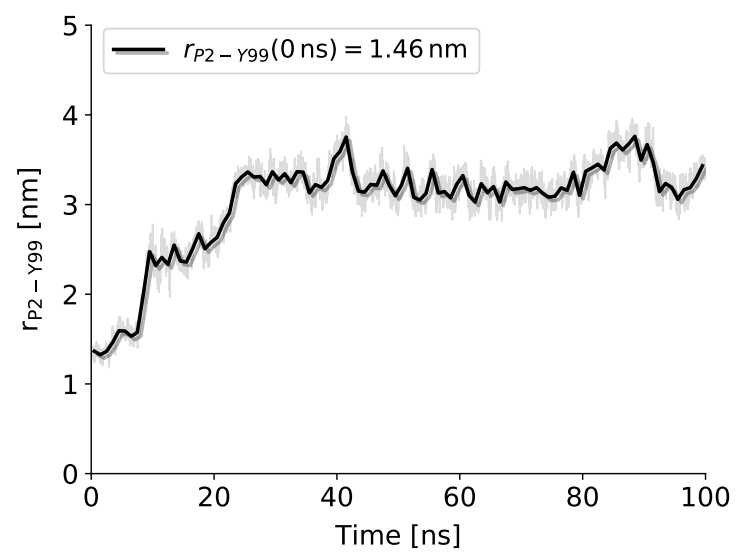

Figure S9: Continued. 
13)

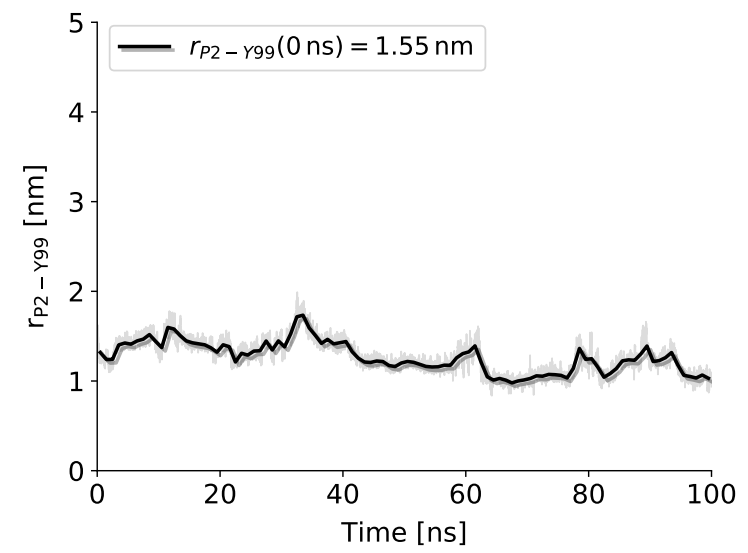

15)

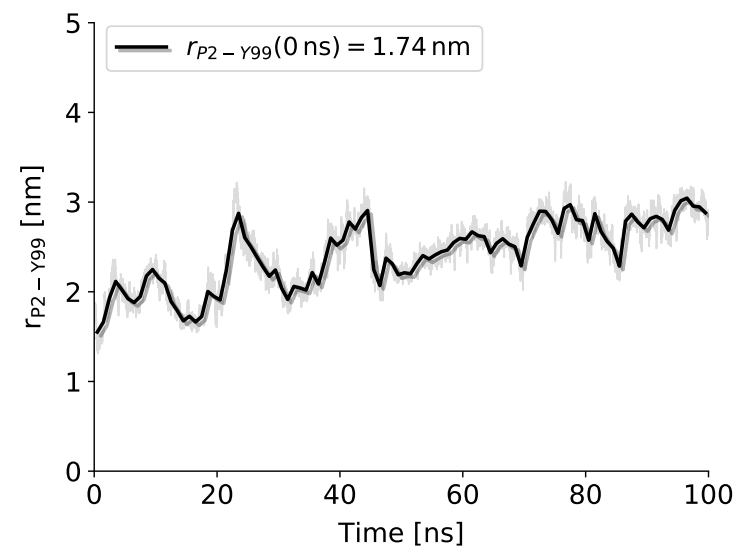

17)

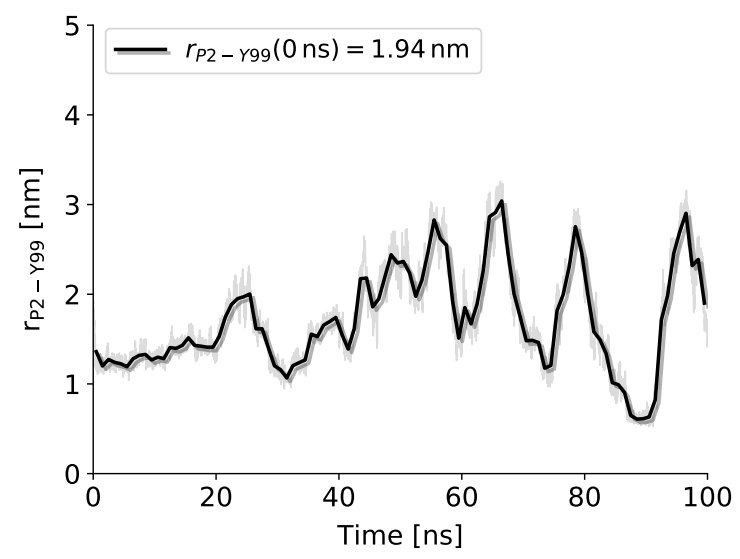

14)

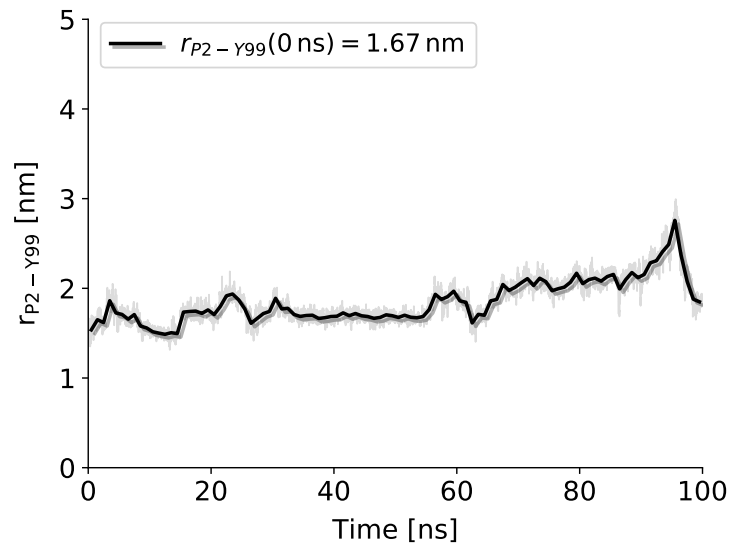

16)

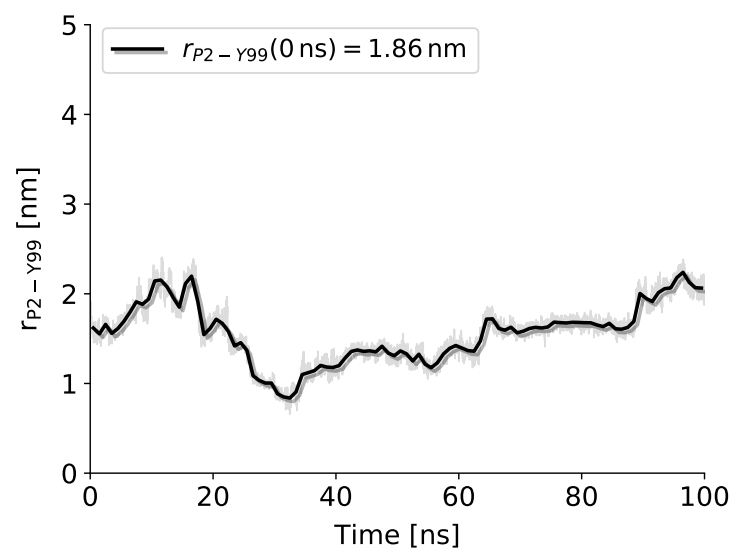

18)

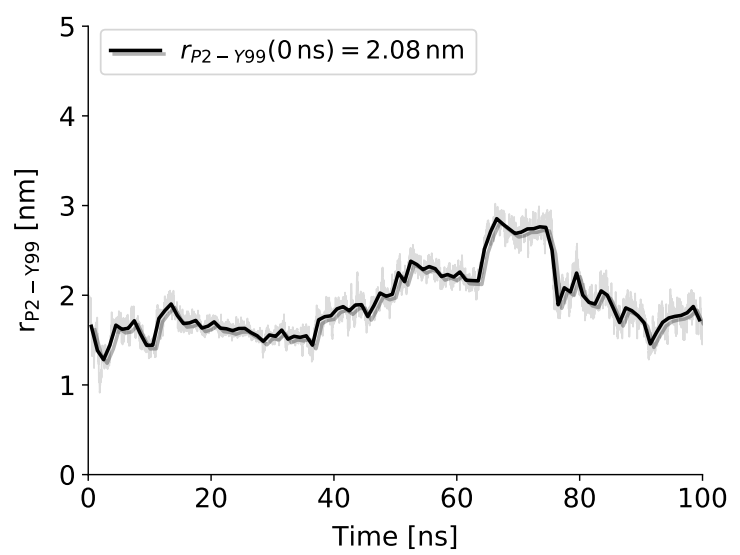

Figure S9: Continued. 
19)

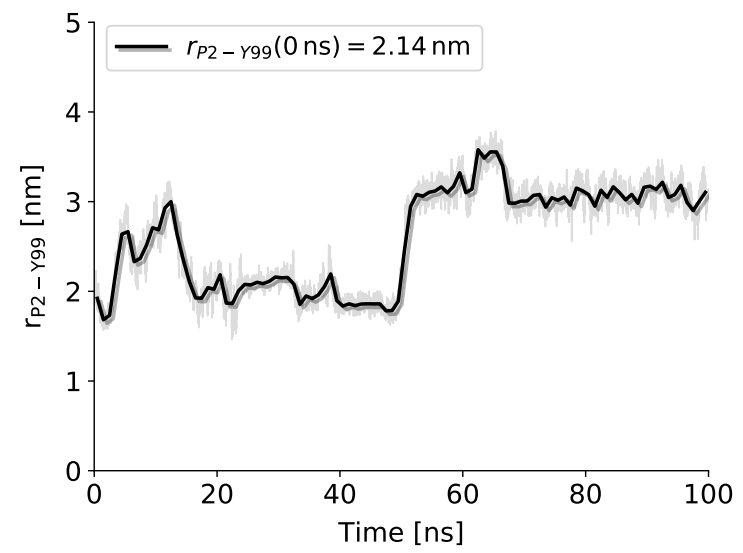

21)

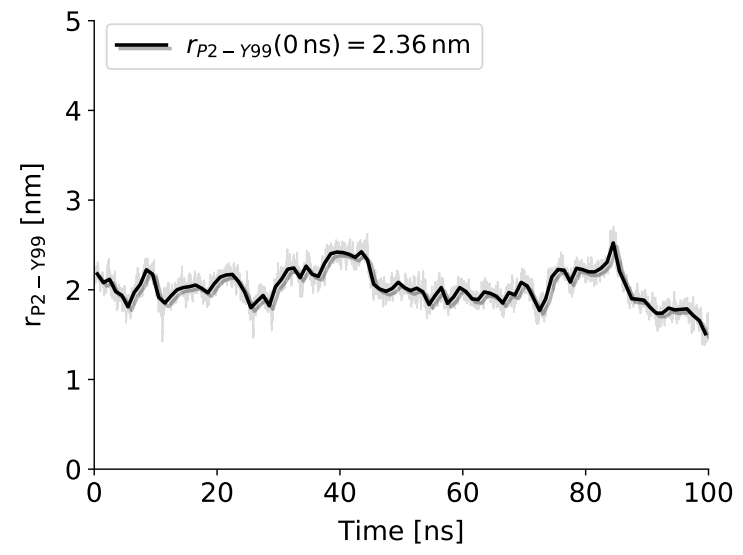

23)

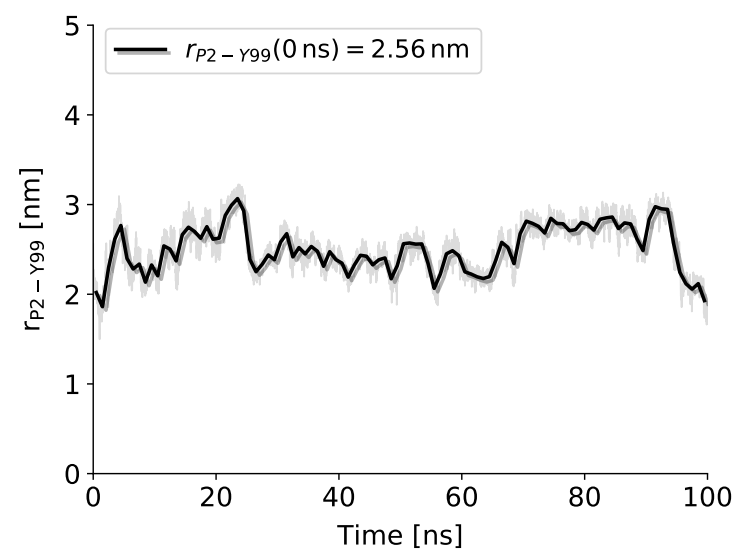

20)

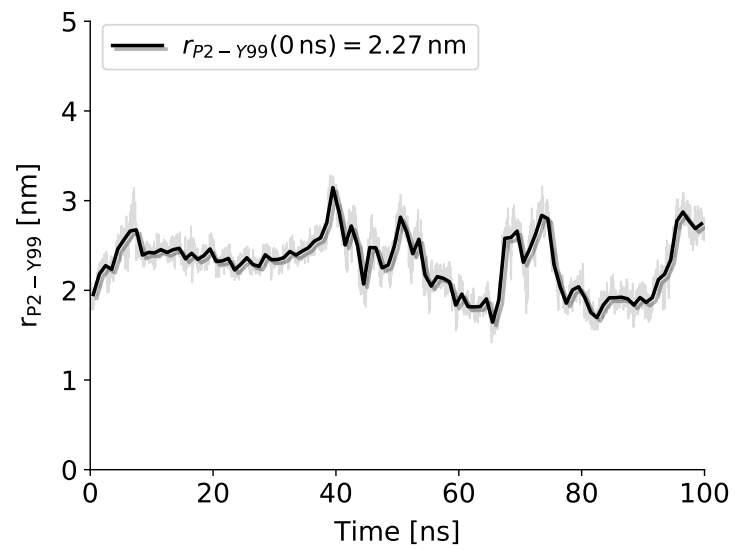

22)

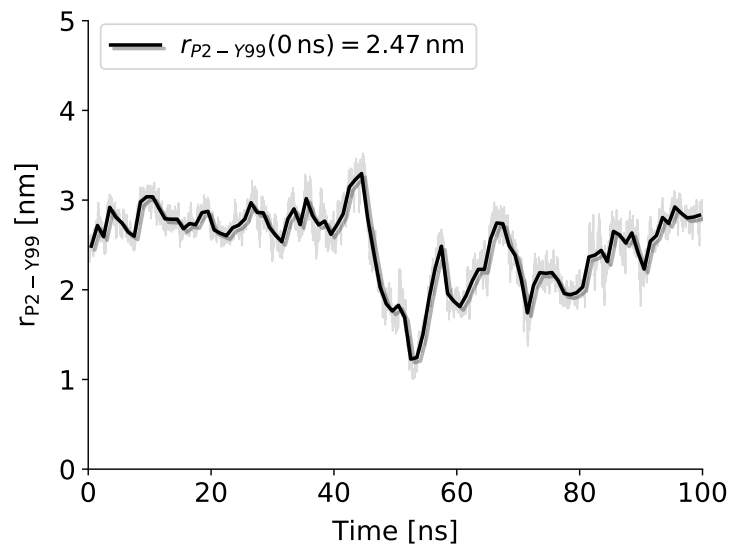

Figure S9: Continued. 


\subsection{Pseudocode for Volume Calculation}

// This program is used to estimate the volume engulfed by the MHC I binding groove.

// It uses the coordinates of the $\mathrm{C}_{\alpha}$-atoms of the binding groove as input.

// Next, it enforces a standard orientation of the binding groove by PCA.

// The binding groove is then filled with small cubes whose volumes are added up.

$$
\begin{aligned}
& \text { constructorGrooveVolumeClass() } \\
& \text { read in coordinates from .pdb file } \\
& \text { doPrincipalComponentAnalysis() } \\
& \text { determineReferencePoints() }
\end{aligned}
$$

doPrincipalComponentAnalysis()

calculate covariance matrix

diagonalise covariance matrix and obtain eigenvectors

// The binding groove is always longer than wide and wider than high.

$/ /=>$ After PCA, the x-axis corresponds to the length, the y-axis to the width,

// and the z-axis to the height of the binding groove.

use eigenvectors to rotate binding groove

shift center of geometry of binding groove to origin

determineReferencePoints()

select parts of the binding groove (Tab. S4)

calculate centers of geometry of the selected parts 
// This function removes cubes that overlay with the binding groove residues.

// The ellipsoids defined in checkForPointsOutsideTheGroove() cannot be positioned // precisely enough to avoid those clashes.

checkForPointsInsideCube()

skipThisIteration $=$ false

loop over all dimensions

lowerBound $=$ centerOfNewCube $-0.5 *$ lengthOfCube

upperBound $=$ centerOfNewCube $+0.5^{*}$ lengthOfCube

loop over all points

if (pointCoordinate $>$ lowerBound and pointCoordinate $<$ upperBound) checkPointSingleCoordinate $=1$

else

checkPointSingleCoordinate $=0$

end loop

end loop

loop over all points

checkPoint $=0$

loop over all dimensions

checkPoint $+=$ checkPointSingleCoordinate

end loop

if $($ checkPoint $==3$ )

skipThisIteration $=$ true

break

end loop

return skipThisIteration 
// This function defines ellipsoids to cover the volume engulfed by the binding groove.

// Points that are not inside any of the ellipsoids are discarded.

checkForPointsOutsideTheGroove()

loop over all centers of geometry

calculate distance between centerOfNewCube and center of geometry

end loop

skipThisIteration $=$ true

// The empirical pre-factors determine the softness and size of the ellipsoids.

if $(1.9 *$ distanceToCogOfBindingGroove $<=$ distanceToCogOfAlpha2Helix)

skipThisIteration $=$ false

else if $\left(2.5^{*}\right.$ distanceToCogOfBindingGroove $<=$ distanceToCogOfAlpha1Helix)

skipThisIteration $=$ false

else if $\left(3.2^{*}\right.$ distanceToCogOfBindingGroove $<=$ distanceToCogOfTop)

skipThisIteration $=$ false

else if $\left(3.7^{*}\right.$ distanceToCogOfBindingGroove $<=$ distanceToCogOfBottom)

skipThisIteration $=$ false

else if $\left(3.8^{*}\right.$ distanceToCogOfTop $<=$ distanceToCogOfBindingGroove)

skipThisIteration $=$ false

else if $\left(4.5^{*}\right.$ distanceToCogOfBottom $<=$ distanceToCogOfBindingGroove)

skipThisIteration $=$ false

else if $\left(2.0^{*}\right.$ distanceToCogOfUpperBindingGroove $<=$

distanceToCogOfUpperAlpha2Helix)

skipThisIteration $=$ false

else if $\left(2.5^{*}\right.$ distanceToCogOfUpperBindingGroove $<=$

distanceToCogOfUpperAlpha1Helix)

skipThisIteration $=$ false 
else if $\left(1.8^{*}\right.$ distanceToCogOfLowerBindingGroove $<=$

distanceToCogOfLowerAlpha2Helix)

skipThisIteration $=$ false

else if $\left(2.1^{*}\right.$ distanceToCogOfLowerBindingGroove $<=$

distanceToCogOfLowerAalpha1Helix)

skipThisIteration $=$ false

else if $\left(2.8^{*}\right.$ distanceToCogOfAlpha2Helix $<=$ distanceToCogOfUpperAlpha2Helix)

skipThisIteration $=$ false

else if $\left(2.8^{*}\right.$ distanceToCogOfAlpha2Helix $<=$ distanceToCogOfLowerAlpha2Helix)

skipThisIteration $=$ false

else if (3.5*distanceToCogOfAlpha1Helix $<=$ distanceToCogOfUpperAlpha1Helix)

skipThisIteration $=$ false

else if $\left(3.5^{*}\right.$ distanceToCogOfAlpha1Helix $<=$ distanceToCogOfLowerAalpha1Helix)

skipThisIteration $=$ false

else if $\left(4.5^{*}\right.$ distanceToCogOfLowerBindingGroove $<=$ distanceToCogOfTop)

skipThisIteration $=$ false

else if $\left(4.6^{*}\right.$ distanceToCogOfUpperBindingGroove $<=$ distanceToCogOfBottom)

skipThisIteration $=$ false

else if $(1.4 *$ distanceToCogOfTop $2<=$ distanceToCogOfUpperBindingGroove)

skipThisIteration $=$ false

else if $\left(1.6^{*}\right.$ distanceToCogOfBottom $2<=$ distanceToCogOfLowerBindingGroove)

skipThisIteration $=$ false

else if $\left(1.8^{*}\right.$ distanceToCogOfBothHelices $<=$ distanceToCogOfAlpha2Helix $)$

skipThisIteration $=$ false

else if $\left(2.2^{*}\right.$ distanceToCogOfBothHelices $<=$ distanceToCogOfAlpha1Helix)

skipThisIteration $=$ false 
else if $\left(3.2^{*}\right.$ distanceToCogOfBothHelices $<=$ distanceToCogOfTop)

skipThisIteration $=$ false

else if $\left(3.7^{*}\right.$ distanceToCogOfBothHelices $<=$ distanceToCogOfBottom $)$

skipThisIteration $=$ false

else if $\left(1.8^{*}\right.$ distanceToCogOfBothUpperHelices $<=$ distanceToCogOfUpperAlpha2Helix)

skipThisIteration $=$ false

else if $\left(2.5^{*}\right.$ distanceToCogOfBothUpperHelices $<=$ distanceToCogOfUpperAlpha1Helix) skipThisIteration $=$ false

else if $\left(1.8^{*}\right.$ distanceToCogOfBothLowerHelices $<=$ distanceToCogOfLowerAlpha2Helix)

skipThisIteration $=$ false

else if $\left(2.3^{*}\right.$ distanceToCogOfBothLowerHelices $<=$ distanceToCogOfLowerAalpha1Helix)

skipThisIteration $=$ false

else if $\left(5.2^{*}\right.$ distanceToCogOfBothLowerHelices $<=$ distanceToCogOfTop)

skipThisIteration $=$ false

else if $(5.0 *$ distanceToCogOfBothUpperHelices $<=$ distanceToCogOfBottom)

skipThisIteration $=$ false

return skipThisIteration 


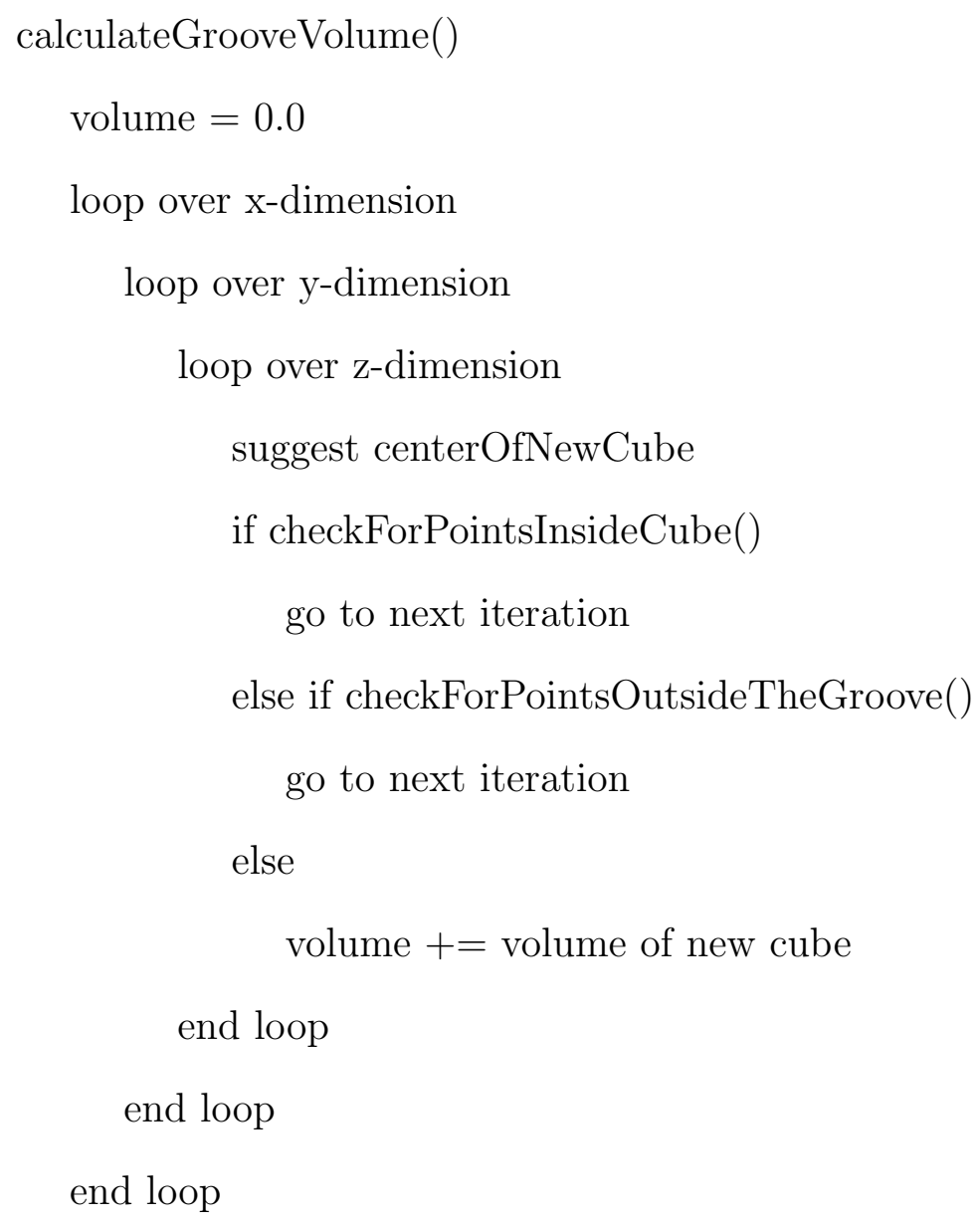


Table S4: List of the centers of geometry used to position the ellipsoids approximating the volume of the binding groove.

\begin{tabular}{ll}
\hline COG name & Selected residues \\
\hline binding groove & $4-11,23-36,46-85,93-101,112-118,122-126,137-180$ \\
upper binding groove & $4-9,23-36,46-70,99-101,159-180$ \\
lower binding groove & $10-11,71-85,93-98,112-118,122-126,137-158$ \\
$\alpha_{1}$-helix & $56-85$ \\
upper $\alpha_{1}$-helix & $56-70$ \\
lower $\alpha_{1}$-helix & $71-85$ \\
$\alpha_{2}$-helix & $137-180$ \\
upper $\alpha_{2}$-helix & $159-180$ \\
lower $\alpha_{2}$-helix & $137-158$ \\
top & $52-59,168-173$ \\
bottom & $80-82,142-144$ \\
top 2 & $28-36,52-55,169-172$ \\
bottom 2 & $80-82,115-121,142-144$ \\
both helices & $56-85,137-180$ \\
both upper helices & $56-70,159-180$ \\
both lower helices & $71-85,137-158$ \\
\hline
\end{tabular}




\section{References}

(1) Cornell, W. D.; Cieplak, P.; Bayly, C. I.; Gould, I. R.; Merz, K. M.; Jr.,; Ferguson, D. M.; Spellmeyer, D. C.; Fox, T.; Caldwell, J. W.; Kollman, P. A. A Second Generation Force Field for the Simulation of Proteins, Nucleic Acids, and Organic Molecules. J. Am. Chem. Soc. 1995, 117, 5179-5197.

(2) Hornak, V.; Abel, R.; Okur, A.; Strockbine, B.; Roitberg, A.; Simmerling, C. Comparison of Multiple Amber Force Fields and Development of Improved Protein Backbone Parameters. Proteins: Struct., Funct., Bioinf. 2006, 65, 712-725.

(3) Best, R. B.; Hummer, G. Optimized Molecular Dynamics Force Fields Applied to the Helix-Coil Transition of Polypeptides. J. Phys. Chem. B 2009, 113, 9004-9015.

(4) Lindorff-Larsen, K.; Piana, S.; Palmo, K.; Maragakis, P.; Klepeis, J. L.; Dror, R. O.; Shaw, D. E. Improved sidechain torsion potentials for the Amber ff99SB protein force field. Proteins: Struct., Funct., Bioinf. 2010, 78, 1950-1958.

(5) Aliev, A. E.; Kulke, M.; Khaneja, H. S.; Chudasama, V.; Sheppard, T. D.; Lanigan, R. M. Motional timescale predictions by molecular dynamics simulations: Case study using proline and hydroxyproline sidechain dynamics. Proteins: Struct., Funct., Bioinf. 2014, 82, 195-215.

(6) Abascal, J. L. F.; Vega, C. A general purpose model for the condensed phases of water: TIP4P/2005. J. Chem. Phys. 2005, 123, 234505.

(7) Abraham, M. J.; Murtola, T.; Schulz, R.; Páll, S.; Smith, J. C.; Hess, B.; Lindahl, E. GROMACS: High performance molecular simulations through multi-level parallelism from laptops to supercomputers. SoftwareX 2015, 1-2, 19-25.

(8) Páll, S.; Abraham, M. J.; Kutzner, C.; Hess, B.; Lindahl, E. In Solving Software Challenges for Exascale; Markidis, S., Laure, E., Eds.; 2015; Vol. 8759; pp 3-27. 
(9) Pronk, S.; Páll, S.; Schulz, R.; Larsson, P.; Bjelkmar, P.; Apostolov, R.; Shirts, M. R.; Smith, J. C.; Kasson, P. M.; van der Spoel, D.; Hess, B.; Lindahl, E. GROMACS 4.5: a high-throughput and highly parallel open source molecular simulation toolkit. Bioinformatics 2013, 29, 845-854.

(10) Hess, B.; Kutzner, C.; van der Spoel, D.; Lindahl, E. GROMACS 4: Algorithms for Highly Efficient, Load-Balanced, and Scalable Molecular Simulation. J. Chem. Theory Comput. 2008, 4, 435-447.

(11) van der Spoel, D.; Lindahl, E.; Hess, B.; Groenhof, G.; Mark, A. E.; Berendsen, H. J. C. GROMACS: Fast, Flexible, and Free. J. Comput. Chem. 2005, 26, 1701-1718.

(12) Lindahl, E.; Hess, B.; van der Spoel, D. GROMACS 3.0: a package for molecular simulation and trajectory analysis. J. Mol. Model. 2001, 7, 306-317.

(13) Berendsen, H. J. C.; van der Spoel, D.; van Drunen, R. GROMACS: A message-passing parallel molecular dynamics implementation. Comp. Phys. Comm. 1995, 91, 43-56.

(14) Torrie, G. M.; Valleau, J. P. Monte Carlo free energy estimates using non-Boltzmann sampling: Application to the sub-critical Lennard-Jones fluid. Chem. Phys. Lett. 1974, 28, 578-581.

(15) Torrie, G. M.; Valleau, J. P. Nonphysical Sampling Distributions in Monte Carlo FreeEnergy Estimation: Umbrella Sampling. J. Comput. Phys. 1977, 23, 187-199.

(16) Kästner, J. Umbrella sampling. WIREs Comput. Mol. Sci. 2011, 1, 932-942.

(17) Sugita, Y.; Kitao, A.; Okamoto, Y. Multidimensional replica-exchange method for freeenergy calculations. J. Chem. Phys. 2000, 113, 6042-6051.

(18) Liu, P.; Kim, B.; Friesner, R. A.; Berne, B. J. Replica exchange with solute tempering: A method for sampling biological systems in explicit water. PNAS 2005, 102, 1374913754 . 
(19) Terakawa, T.; Kameda, T.; Takada, S. On Easy Implementation of a Variant of the Replica Exchange with Solute Tempering in GROMACS. J. Comput. Chem. 2011, 32, $1228-1234$.

(20) Wang, L.; Friesner, R. A.; Berne, B. J. Replica Exchange with Solute Scaling: A More Efficient Version of Replica Exchange with Solute Tempering (REST2). J. Phys. Chem. B 2011, 115, 9431-9438.

(21) Barducci, A.; Bussi, G.; Parrinello, M. Well-Tempered Metadynamics: A Smoothly Converging and Tunable Free-Energy Method. Phys. Rev. Lett. 2008, 100, 020603.

(22) Sugita, Y.; Okamoto, Y. Replica-exchange molecular dynamics method for protein folding. Chem. Phys. Lett. 1999, 314, 141-151.

(23) Tribello, G. A.; Bonomi, M.; Branduardi, D.; Camilloni, C.; Bussi, G. PLUMED 2: New feathers for an old bird. Comput. Phys. Commun. 2014, 185, 604-613.

(24) Hess, B.; Bekker, H.; Berendsen, H. J. C.; Fraaije, J. G. E. M. LINCS: A Linear Constraint Solver for Molecular Simulations. J. Comput. Chem. 1997, 18, 1463-1472.

(25) Hess, B. P-LINCS: A Parallel Linear Constraint Solver for molecular simulation. J. Chem. Theory Comput. 2008, 4, 116-122.

(26) Miyamoto, S.; Kollman, P. A. SETTLE: An Analytical Version of the SHAKE and RATTLE Algorithm for Rigid Water Models. J. Comput. Chem. 1992, 13, 952-962.

(27) Essmann, U.; Perera, L.; Berkowitz, M. L.; Darden, T.; Lee, H.; Pedersen, L. G. A smooth particle mesh Ewald method. J. Chem. Phys. 1995, 103, 8577-8593.

(28) Smit, B.; Frenkel, D. Vapor-liquid equilibria of the two-dimensional Lennard-Jones fluid(s). J. Chem. Phys. 1991, 94, 5663-5668. 
(29) Kumar, S.; Bouzida, D.; Swendsen, R. H.; Kollman, P. A.; Rosenberg, J. M. The Weighted Histogram Analysis Method for Free-Energy Calculations on Biomolecules. I. The Method. J. Comput. Chem. 1992, 13, 1011-1021.

(30) Hub, J. S.; de Groot, B. L.; van der Spoel, D. g_wham - A Free Weighted Histogram Analysis Implementation Including Robust Error and Autocorrelation Estimates. J. Chem. Theory Comput. 2010, 6, 3713-3720.

(31) Schlitter, J. Estimation of absolute and relative entropies of macromolecules using the covariance matrix. Chem. Phys. Lett. 1993, 215, 617-621. 